\title{
Operator norm theory as an efficient tool to propagate hybrid uncertainties and calculate imprecise probabilities
}

\author{
Matthias G.R. Faes ${ }^{\mathrm{a}, \mathrm{c}}$, Marcos A. Valdebenito ${ }^{\mathrm{b}}$, David Moens ${ }^{\mathrm{a}}$, Michael Beer ${ }^{\mathrm{c}, \mathrm{d}, \mathrm{e}}$ \\ ${ }^{a}$ KU Leuven, Department of Mechanical Engineering, Technology campus De Nayer, Jan De Nayerlaan 5, St.-Katelijne-Waver, \\ Belgium \\ ${ }^{b}$ Universidad Tecnica Federico Santa Maria, Dept. de Obras Civiles, Av. España 1680, Valparaiso, Chile \\ ${ }^{c}$ Institute for Risk and Reliability, Leibniz Universität Hannover, Callinstr. 34, 30167 Hannover, Germany \\ ${ }^{d}$ Institute for Risk and Uncertainty and School of Engineering, University of Liverpool, Peach Street, Liverpool L69 7ZF, UK \\ ${ }^{e}$ International Joint Research Center for Engineering Reliability and Stochastic Mechanics, Tongji University, 1239 Siping \\ Road, Shanghai 200092, P.R. China
}

\begin{abstract}
1 This paper presents a highly efficient and effective approach to bound the responses and probability of failure 2 of linear systems where the model parameters are subjected to combinations of epistemic and aleatory uncer3 tainty. These combinations can take the form of imprecise probabilities or hybrid uncertainties. Typically, 4 such computations involve solving a nested double loop problem, where the propagation of the aleatory 5 uncertainty has to be performed for each realisation of the epistemic uncertainty. Apart from near-trivial 6 cases, such computation is intractable without resorting to surrogate modeling schemes. In this paper, a 7 method is presented to break this double loop by virtue of the operator norm theorem. Indeed, in case lin8 ear models are considered and under the restriction that the model definition cannot be subject to aleatory 9 uncertainty, the paper shows that the computational efficiency, quantified by the required number of model

evaluations, of propagating these parametric uncertainties can be improved by several orders of magnitude. Two case studies involving a finite element model of a clamped plate and a six-story building are included to illustrate the application of the developed technique, as well as its computational merit in comparison to existing double-loop approaches.
\end{abstract}

Keywords: Uncertainty Quantification, imprecise probabilities, operator norm theorem, linear models, decoupling

\section{Introduction}

Many of the current advanced modelling approaches in an engineering context rely heavily on problems formulated over a continuum domain, which is also referred to as continuum physics. Typical examples include material deformation in an elastic continuum under external loading conditions for the calculation of strains and stresses over a spatial domain (= continuum mechanics), the assessment of the dynamical response of such continuum to external loads (= structural dynamics) or fluid flow analysis in a spatial continuum for the study of local particle velocities or fluid pressure values (= computational fluid dynamics). 
Once the fundamental constitutive equations and required boundary conditions are formulated over the considered continuum, the numerical modelling strategy typically consists of discretising the continuous domain over time and/or space, after which the discretised version of the problem is formulated in a finitedimensional system of equations (e.g., using finite elements or volumes). Many modelling approaches based on this principal idea have been developed and extensively refined over the past decades, resulting in several commercial codes and wide academic and industrial application. However, it is more and more acknowledged that one deterministic 'nominal' analysis (i.e., under the assumption of full and deterministic knowledge on any property appearing at any time and location in the continuum) is often insufficient to fully assess the problem at hand. Especially from an engineering perspective, correctly assessing the uncertainty in the analysis outcome is of crucial importance to ensure reliability of a designed structure or component. This motivates the use of non-deterministic modelling techniques to account for potential lack-of-knowledge in both the model form (i.e., the application of the correct equations in the continuum problem) as well its parameters. In this manuscript, only the case of parametric uncertainty is further considered.

In the context of performing non-deterministic analysis, several parameters and inputs of the continuum that is modelled have to be represented by an uncertainty model. This model represents a predefined description of the inherent variability, vagueness, ambiguity, lack of knowledge or a combination of these factors into a mathematically rigorous framework that allows for eliciting the uncertainty in the response of the continuum under consideration. Several categories of parameters and inputs can be defined in this context, based on their origin as aleatory uncertainty (inherent variation) or/and epistemic uncertainty (lack of knowledge) [1]:

Type I: Parameters without any uncertainty, modelled as a crisp number

Type II: Parameters containing only epistemic uncertainty, appearing as unknown-but-fixed constants or variable properties

Type III: Parameters containing only aleatory uncertainty, appearing as random variables with a fully prescribed stochastic description

Type IV: Parameters containing both aleatory and epistemic uncertainties, represented as imprecise probabilities

The correct approach to deal with these types of non-determinism is inherently linked with their definition. The modelling and simulation of Type-II uncertain parameters typically is performed via the framework of interval or fuzzy analysis [2]. For the remainder of this paper, a Type-II uncertain parameter $\theta \in \theta_{I I}$ is represented as belonging to an interval $\theta^{I}$, i.e., $\theta \in \theta^{I I}:=\theta^{I}=[\underline{\theta}, \bar{\theta}]$, where $\underline{\theta}$ and $\bar{\theta}$ represent the lower and upper bounds. In other words, the uncertainty an analyst has concerning the true value of $\theta$ is 
translated towards fixed bounds between which the valued is deemed to lie, without assigning a likelihood to any value within these bounds [3]. The main advantage of this class of methods is that intervals require only very few data points to make an objective worst-case estimate of the bounds on the structural behavior of the model under consideration, conditional on the data [4]. Furthermore, recent developments allow for estimating robust interval bounds given only limited data, for instance based on worst-case likelihood estimation (see e.g., $[5,6]$ ), or Chebyshev's inequality. As a drawback, intervals provide only the worst and best case structural response to the analyst $[7,8]$. Furthermore, the modelling of dependencies between uncertain parameters requires dedicated methods based on the projection of $\theta^{I}$ to a non-orthogonal basis [9], the admissible set decomposition method [10] or using affine arithmetic [11]. Alternatively, also convex set approaches can be applied to represent Type-II uncertain properties [12].

The variability in type-III uncertain parameters is usually characterized by a probability density function $f_{\Phi}(\phi)$ that represents the likelihood that a parameter $\phi$ assumes a certain value within a specified range. To infer the likelihood that the model assumes a certain response, given this uncertain parameter or input, $f_{\Phi}(\phi)$ is propagated through the model. A vast literature amount of literature exists on computing central moments or expected values of a response of interest of the model, based on $f_{\Phi}(\phi)$ [13]. Recent developments in this context include advanced sampling schemes such as multilevel approaches [14], Subset simulation [15] or importance sampling $[16,17]$, techniques based on surrogate modelling $[18,19]$ or stochastic linearization $[20$, 21]. A good overview of stochastic methods in engineering applications is given by [22].

Type-IV uncertainties, which are used to represent deep uncertainties (sometimes also referred to as polymorphic [23]) can be defined using several highly advanced modelling techniques, including probability boxes [24], Evidence theory [25] or possibility functions [26]. The most straightforward way to model an imprecise probability is probably using interval or fuzzy probabilities [27], where intervals (or fuzzy numbers) are assigned to the central moments of a type-III uncertain parameter. Recent advances in propagation algorithms include methods based on Polynomial Chaos Expansions [28, 29], interval predictor models [30], methods based on importance sampling [31] potentially in combination with a high-dimensional model representation of the underlying numerical model $[32,19]$, techniques based on affine arithmetic [33] or multi-level strategies [34]. Furthermore, also efficient interval Monte Carlo [35, 36] or techniques based on linear programming [37] have been introduced in this context. Finally, also highly performing methods for performing state-estimation of nonlinear systems based on Kalman filtering have been recently introduced [38]. A good recent overview of imprecise probabilistic approaches is given by [39] or by [40] on the topic of hybrid analysis. Recent applications include the study of the uncertainty in the mechanical properties of wood [41], assessing the effect of geometric imperfections in cylindrical shells [42], structural reliability analysis [43] or real-time predictions of mechanised tunneling processes [44]. 
However, most methods to propagate type-IV uncertain properties still rely on some sort of double loop approach where the propagation of the aleatory part of the uncertainty has to be performed for each realization of the epistemic uncertainty, or vice-versa. Apart from the case where near-trivial simulation methods are considered, this impedes their application without resorting to surrogate modelling. Despite the fact that these approaches are highly performing, they still rely on an approximate relation between input and output. This contribution elaborates on previous work of the authors [45], where a highly efficient approach to decouple the "double-loop" based on operator norm theory was presented in the context of linear structures that are excited with a zero-mean imprecisely defined stochastic ground acceleration. This paper extends these developments to (1) account for non-homogeneous loads and (2) include epistemic uncertainty in the structural model itself, hence making it applicable to both the case where a model is subjected to a type-IV load (i.e., an imprecise probability) or combinations of Type-II, Type-III and Type-IV uncertainties (i.e., hybrid uncertainty). The results show that also in these cases, a method based on operator norm theory is capable of successfully decoupling the double-loop, resulting in a gain in computational efficiency of several orders of magnitude compared to traditional double-loop methods, as evidenced by the significant decrease in required number of model evaluations in the included case studies. The paper is structured as follows: Section 2 discusses the background behind imprecise probabilistic analysis with linear models; Section 3 presents the operator norm framework to efficiently propagate imprecise probabilities; Section 4 shows two numerical examples involving a finite element model of a clamped plate, as well as a 6-story building; Section 5 lists the conclusions of the work.

\section{Imprecise probabilistic analysis}

\subsection{Model definition}

The main idea to fully decouple the propagation of epistemic and aleatory uncertainty is based on previous work of the authors [45], where they showed that operator norm theory can be used to successfully decouple the epistemic and aleatory uncertainty of the response associated with a crisp, linear structural model subject to imprecise stochastic loading (that is, loading described by means of Type-IV uncertainty). The current contribution expands on the type of problems considered in the aforementioned work. In particular, the scope of application of the method is on models whose response can be cast in the following form:

$$
\boldsymbol{y}(\boldsymbol{\theta}, \vartheta, \boldsymbol{z})=\boldsymbol{A}(\boldsymbol{\theta}) \boldsymbol{z}(\boldsymbol{\vartheta})
$$

with $\boldsymbol{y} \in \mathbb{R}^{d_{y}}$ the response of the model under consideration (e.g., a mechanical stress or temperature distribution), $\boldsymbol{\theta} \in \mathcal{T} \subset \mathbb{R}^{d_{\theta}}$ a vector of model parameters (e.g., a constitutive material model or the description of a boundary condition) belonging to an admissible set $\mathcal{T}$ (e.g., non-negative stiffness values or temperatures). 
$\boldsymbol{A}: \mathbb{R}^{d_{z}} \mapsto \mathbb{R}^{d_{y}}$ is herein a linear map that describes the physical behaviour of the continuum that is being modelled, which is in an engineering context often represented via a finite element or finite difference model. For example, within the framework of structural mechanics, $\boldsymbol{A}$ corresponds to the inverse of the stiffness matrix. The parameter $\boldsymbol{z}$ is the input to the model (e.g., a distributed load, pressure distribution or thermal flux) and is parameterized by a vector $\vartheta \in \mathbb{R}^{d_{\vartheta}}$. In essence, the model form in Eq. (1) corresponds to the class of linear models that represent a discretized formulation of the continuum physics at hand (e.g., finite element models), that are furthermore subjected to a stochastic process load that can be recast in a series expansion form that allows separating the stochastic content from the temporal dependence, such as for instance the Karhunen-Loève series expansion (see Appendix A).

\subsection{Crisp Failure Probability}

In a classical reliability engineering context, the analyst is interested in computing the probability of failure $P_{f}$ of the structure given a predefined type-III uncertainty in its definition or the imposed load condition. To account for such aleatory uncertainty, the model introduced in Eq. (1) becomes:

$$
\boldsymbol{y}\left(\boldsymbol{\theta}_{I}, \boldsymbol{\vartheta}_{I}, \boldsymbol{x}_{I I I}\right)=\boldsymbol{A}\left(\phi_{I I I}, \boldsymbol{\theta}_{I}\right) \boldsymbol{z}\left(\boldsymbol{\xi}_{I I I}, \boldsymbol{\vartheta}_{I}\right),
$$

with $\boldsymbol{x}=[\boldsymbol{\phi}, \boldsymbol{\xi}]$, with $\boldsymbol{\phi} \in \mathbb{R}^{d_{\phi}}$ and $\boldsymbol{\xi} \in \mathbb{R}^{d_{\xi}}$, and where $\boldsymbol{\phi}_{I I I}$ and $\boldsymbol{\xi}_{I I I}$ represent type-III uncertain properties of the structural model and the model input, respectively. These properties are represented by their respective probability density functions $f_{\boldsymbol{\Phi}}(\boldsymbol{\phi})$ and $f_{\boldsymbol{\Xi}}(\boldsymbol{\xi})$. In this case, the probability of failure $P_{f}$ is readily defined as:

$$
P_{f}=\int_{\boldsymbol{x} \in \mathbb{R}^{d_{x}}} I_{F}(\boldsymbol{x}) f_{\boldsymbol{X}}(\boldsymbol{x}) d \boldsymbol{x}
$$

where $f_{\boldsymbol{X}}(\cdot)$ is the joint distribution function of $f_{\boldsymbol{\Phi}}(\boldsymbol{\phi})$ and $f_{\boldsymbol{\Xi}}(\boldsymbol{\xi})$ in $d_{x}=d_{\phi}+d_{\xi}$ dimensions, and $I_{F}(\cdot)$ is an indicator function whose value is equal to one in case of a failure event and zero otherwise:

$$
I_{F}= \begin{cases}1 & y_{i}\left(\boldsymbol{\theta}_{I}, \boldsymbol{\vartheta}_{I}, \boldsymbol{x}_{I I I}\right) \geq y_{t} i=1, \ldots, d_{y} \\ 0 & \text { otherwise }\end{cases}
$$

with $y_{t}$ a predefined threshold value, corresponding to a structural failure. It should be noted that the probability integral in Eq. (3) usually comprises a high number of dimensions, as $n_{x}$ may be in the order of hundreds or thousands, while the performance function is known point-wise only for specific realizations $\boldsymbol{x}$ of $\boldsymbol{X}$. This precludes the application of quadrature schemes for evaluating $P_{f}$ and favor the application of simulation methods, see e.g. [46]. However, also the application of simulation methods can be computationally 
costly since repeated evaluations of Eq. (1) are required.

\subsection{Imprecise failure probability}

The computation of a crisp failure probability requires the exact definition of the probability density functions $f_{\boldsymbol{\Phi}}(\boldsymbol{\phi})$ and $f_{\boldsymbol{\Xi}}(\boldsymbol{\xi})$. However, in many real life situations, the analyst only has partial information about these quantities. As a result, also type-II and type-IV uncertainties have to be taken into account during the modelling phase. To account for various sources of uncertainty in the description of the different model quantities, as described in the introduction, the description of the model that is introduced in Eq. (1) is as such extended as:

$$
\boldsymbol{y}\left(\boldsymbol{\theta}_{I I}, \boldsymbol{\vartheta}_{I I}, \phi_{I I I}, \boldsymbol{\xi}_{I I I}\right)=\boldsymbol{A}\left(\phi_{I I I}, \boldsymbol{\theta}_{I I}\right) \boldsymbol{z}\left(\boldsymbol{\xi}_{I I I}, \boldsymbol{\vartheta}_{I I}\right)
$$

where the index ${ }_{I I}$ in $\boldsymbol{\theta}$ and $\boldsymbol{\vartheta}$ denote that these parameters are subjected to epistemic uncertainty, modelled for instance as intervals $\boldsymbol{\theta}^{I}$ and $\boldsymbol{\vartheta}^{I}$ or fuzzy sets. Please note that $\boldsymbol{y}$ is in effect a type-IV uncertain parameter due to the presence of both epistemic (type-II) and aleatory (type-III) uncertain parameters. Note furthermore that in the notation followed in this equation, the implicit assumption is made that a type-IV uncertain parameter (e.g., a Normal random variable prescribed by an interval-valued mean and standard deviation) can be separated into an epistemic and aleatory part. This assumption is warranted when the distribution can be recast into an affine form depending on its defining parameters, as is the case for many commonly used density functions, or when the quantity is prescribed by a random field via the Karhunen-Loève series expansion (see also Appendix A).

Furthermore, since $\boldsymbol{z}$ and $\boldsymbol{A}$ are a function of both a Type II and a Type III valued parameter, they become Type IV valued by construction. As an example, the case presented in Eq. (5) can correspond to a model of a structure that is excited by a load which is governed by both a stochastic and an interval component (such as e.g., an imprecise stochastic process as described in [47]) where some parameters of the model are also described by either stochastic parameters, intervals or a combination of both.

In the more general case of models described by Eq. (5), the definition of a crisp probability of failure is no longer possible. In this case, due to the effect of the type-II uncertain parameters $\boldsymbol{\theta}_{I I}$ and $\boldsymbol{\vartheta}_{I I}, P_{f}$ becomes a type-II uncertainty as well. In case the type-II uncertainties are modelled as intervals, the two following optimization problems over the set $\left\{\boldsymbol{\theta}_{I I} ; \boldsymbol{\vartheta}_{I I}\right\}$ have to be jointly considered to bound $P_{f}$ :

$$
\begin{aligned}
\underline{P}_{f} & =\min _{\boldsymbol{\theta}_{I I}, \boldsymbol{\vartheta}_{I I}}\left(P_{F}\left(\boldsymbol{\theta}_{I I}, \boldsymbol{\vartheta}_{I I}\right)\right) \\
& =\min _{\boldsymbol{\theta}_{I I}, \boldsymbol{\vartheta}_{I I}}\left(\int_{\boldsymbol{x} \in \mathbb{R}^{n_{x}}} I_{F}\left(\boldsymbol{x}_{I I I}, \boldsymbol{\theta}_{I I}\right) f_{\boldsymbol{X}}\left(\boldsymbol{x}_{I I I}, \boldsymbol{\vartheta}_{I I}\right) d \boldsymbol{x}\right),
\end{aligned}
$$


to determine the lower bound of the probability of failure, and

$$
\begin{aligned}
\bar{P}_{f} & =\max _{\boldsymbol{\theta}_{I I}, \boldsymbol{\vartheta}_{I I}}\left(P_{F}\left(\boldsymbol{\theta}_{I I}, \boldsymbol{\vartheta}_{I I}\right)\right) \\
& =\max _{\boldsymbol{\theta}_{I I}, \boldsymbol{\vartheta}_{I I}}\left(\int_{\boldsymbol{x} \in \mathbb{R}^{n_{x}}} I_{F}\left(\boldsymbol{x}_{I I I}, \boldsymbol{\theta}_{I I}\right) f_{\boldsymbol{X}}\left(\boldsymbol{x}_{I I I}, \boldsymbol{\vartheta}_{I I}\right) d \boldsymbol{z}\right),
\end{aligned}
$$

to determine the upper bound. During each step of these optimizations, a full computation of $P_{f}$ has to be performed according to Eq. (3) for each crisp value of $\boldsymbol{\theta} \in \boldsymbol{\theta}_{I I}$ and $\boldsymbol{\vartheta} \in \boldsymbol{\vartheta}_{I I}$. As a side remark, it should be noted that in case fuzzy sets are applied to represent the type-II uncertainty, a third layer is added to the double loop, as a fuzzy set is usually decomposed into a set of intervals according to the $\alpha$-level optimization method [2].

\section{Operator norm theory to propagate Imprecise Probabilistic uncertainty}

As is clear from the previous section, the propagation of imprecise probabilities through a numerical model to infer bounds on the probability of failure usually comprises a high computational cost due to the associated double loop problem. This section presents an approach to efficiently and effectively decouple the aleatory from the epistemic uncertainty, and hence, break the double loop associated with solving Eq. (6) and Eq. (8) by virtue of operator norm theory. This section deepens the theory behind this development and generalizes the approach that was presented in [45] to account for epistemic uncertainty in the structural model definition, as well as non-homogeneous loading conditions.

\subsection{The operator norm: theoretical aspects}

Let $\boldsymbol{D}: \mathbb{R}^{d_{v}} \mapsto \mathbb{R}^{d_{r}}$ be a continuous linear map between two normed vector spaces $\mathbb{R}^{d_{v}}$ and $\mathbb{R}^{d_{r}}$ and $\|\bullet\|_{p^{(i)}}$ be a particular $\mathcal{L}_{p^{(i)}}$ norm on these vector spaces with $i \in[1, \infty)$, then there exist a number $c \in \mathbb{R}$ and vector $\boldsymbol{v} \in \mathbb{R}^{d_{v}}$ such that following inequality always holds:

$$
\|\boldsymbol{D} \boldsymbol{v}\|_{p^{(1)}} \leq|c| \cdot\|\boldsymbol{v}\|_{p^{(2)}},
$$

where $\|\boldsymbol{v}\|_{p^{(i)}}$ is constructed according:

$$
\|\boldsymbol{v}\|_{p^{(i)}}=\left(\sum_{i=1}^{d_{v}}\left|v_{i}\right|^{p^{(i)}}\right)^{1 / p^{(i)}}
$$

with $v_{i} \in \boldsymbol{v}$ and where $|\bullet|$ denotes the absolute value of $\bullet$.

The linear operator $\boldsymbol{D}$ maps the input vector $\boldsymbol{v}$ to an output vector $\boldsymbol{r}$, that is $\boldsymbol{r}=\boldsymbol{D} \boldsymbol{v}$. Such map has a clear analogy with Eq. (1), where $\boldsymbol{A}$ maps the model input $\boldsymbol{z}$ to its response $\boldsymbol{y}$ (i.e., the numerical model of 
the continuum under consideration). As an example and to clarify this point, substitution of Eq. (1) into Eq. (10) gives:

$$
\|\boldsymbol{A} \boldsymbol{z}\|_{p^{(1)}} \leq|c| \cdot|| \boldsymbol{z} \|_{p^{(2)}},
$$

and hence:

$$
\|\boldsymbol{y}\|_{p^{(1)}} \leq|c| \cdot\|\boldsymbol{z}\|_{p^{(2)}} .
$$

Note that for the sake of notational simplicity, the dependence of the model quantities on the various parameters is omitted at this point and $z$ is used to indicate a general input to the model. Physically speaking, these equations state that the length of the uncertain model input $\boldsymbol{z}$, quantified via a pre-described $\mathcal{L}_{p^{(i)}}$-norm, can be increased in the maximal case with a factor $c$ when applying the linear mapping described in Eq. (1). While Eqs. (12) and (13) reveal a clear physical connection between linear maps $\boldsymbol{D}$ and $\boldsymbol{A}$, it should be noted that in more general cases, they are not necessarily equal, as discussed in the forthcoming sections.

A measure for how much a certain deterministic linear map $\boldsymbol{D}$ increases the length of the uncertain model input $\boldsymbol{v}$ in the maximum case, is given by the operator norm $\|\boldsymbol{D}\|_{p^{(1)}, p^{(2)}}$, which is defined in a deterministic sense (i.e., for one realization of the uncertain parameters) as:

$$
\|\boldsymbol{D}\|_{p^{(1)}, p^{(2)}}=\inf \left\{c \geq 0:\|\boldsymbol{D} \boldsymbol{v}\|_{p^{(1)}} \leq|c| \cdot\|\boldsymbol{v}\|_{p^{(2)}} \quad \forall \boldsymbol{v} \in \mathbb{R}^{n_{v}}\right\}
$$

or equivalently:

$$
\|\boldsymbol{D}\|_{p^{(1)}, p^{(2)}}=\sup \left\{\frac{\|\boldsymbol{D} \boldsymbol{v}\|_{p^{(1)}}}{\|\boldsymbol{v}\|_{p^{(2)}}}: \boldsymbol{v} \in \mathbb{R}^{n_{v}} \text { with } \boldsymbol{v} \neq 0\right\} \text {. }
$$

The calculation of a particular $\|\boldsymbol{D}\|_{p^{(1)}, p^{(2)}}$ norm evidently depends on the particular choice of $p^{(1)}$ and $p^{(2)}$. An overview of different operator norm formulations, given $p^{(1)}$ and $p^{(2)}$, is given in Table 1 (taken

\begin{tabular}{|c|c|c|c|}
\hline & $\mathcal{L}_{1}$ & $\mathcal{L}_{2}$ & $\mathcal{L}_{\infty}$ \\
\hline $\mathcal{L}_{1}$ & $\begin{array}{l}\text { Maximum } \mathcal{L}_{1} \text { norm of a } \\
\text { column of } \boldsymbol{D}\end{array}$ & $\begin{array}{l}\text { Maximum } \mathcal{L}_{2} \text { norm of a } \\
\text { column of } \boldsymbol{D}\end{array}$ & $\begin{array}{l}\text { Maximum absolute value } \\
\text { of } \boldsymbol{D}\end{array}$ \\
\hline $\mathcal{L}_{2}$ & NP-hard & $\begin{array}{l}\text { Maximum singular value of } \\
D\end{array}$ & $\begin{array}{l}\text { Maximum } \mathcal{L}_{2} \text { norm of a } \\
\text { row of } \boldsymbol{D}\end{array}$ \\
\hline $\mathcal{L}_{\infty}$ & NP-hard & NP-hard & $\begin{array}{l}\text { Maximum } \mathcal{L}_{1} \text { norm of a } \\
\text { row of } \boldsymbol{D}\end{array}$ \\
\hline
\end{tabular}
from [48]). The columns in this matrix indicate the $\mathcal{L}_{p}$ norm on the domain of $\boldsymbol{D}$, whereas the rows indicate the norm on its co-domain.

Table 1: Formulations for commonly applied operator norms 


\subsection{The operator norm: bounding imprecise failure probabilities}

As indicated in Eq. (6) and Eq. (8), a double loop approach is required when computing the bounds on the probability of failure of a model that is subjected to combinations of Type-II, Type-III and Type-IV uncertainty. However, the preceding discussion shows that for linear, continuous models, a measure exists that represents the magnitude with which a certain model input is amplified towards the output. This notion allows us to decouple Eq. (6) and Eq. (8). For that purpose, assume that Eq. (5) can be recast as:

$$
\boldsymbol{y}\left(\boldsymbol{\theta}_{I I}, \boldsymbol{\vartheta}_{I I}, \boldsymbol{z}_{I V}\right)=\boldsymbol{D}\left(\boldsymbol{\theta}_{I I}, \boldsymbol{\vartheta}_{I I}\right) \boldsymbol{e}\left(\boldsymbol{x}_{I I I}\right),
$$

By virtue of the operator norm theorem, decoupling is applied by first determining those realisations $\boldsymbol{\theta}^{*}, \boldsymbol{\vartheta}^{*}$ of the epistemically uncertain parameters that provide the extrema in terms of the amplification of the input (which according to Eq. (16), correspond to $\boldsymbol{e}$ ) to $\boldsymbol{y}$ and hence, $P_{f}$. These realisations are determined by looking for those realisations of $\boldsymbol{\theta}$ and $\boldsymbol{\vartheta}$ that yield an extremum in the operator norm $\|\boldsymbol{D}\|_{p^{(1)}, p^{(2)}}$ by solving following optimization problems:

$$
\boldsymbol{\theta}_{I I}^{*}, \boldsymbol{\vartheta}_{I I}^{*}=\underset{\boldsymbol{\theta} \in \boldsymbol{\theta}_{I I}, \boldsymbol{\vartheta} \in \boldsymbol{\vartheta}_{I I}}{\operatorname{argmin}}\|\boldsymbol{D}(\boldsymbol{\theta}, \boldsymbol{\vartheta})\|_{p^{(1)}, p^{(2)}}
$$

and

$$
\boldsymbol{\theta}_{I I}^{\bar{*}}, \boldsymbol{\vartheta}_{I I}^{\bar{*}}=\underset{\boldsymbol{\theta} \in \boldsymbol{\theta}_{I I}, \boldsymbol{\vartheta} \in \boldsymbol{\vartheta}_{I I}}{\operatorname{argmax}}\|\boldsymbol{D}(\boldsymbol{\theta}, \boldsymbol{\vartheta})\|_{p^{(1)}, p^{(2)}}
$$

As such, Eq. (6) and Eq. (8) can be reformulated as:

$$
\begin{aligned}
\underline{P}_{f} & =P_{F}\left(\boldsymbol{\theta}_{I I}^{*}, \boldsymbol{\vartheta}_{I I}^{*}\right) \\
& =\int_{\boldsymbol{x} \in \mathbb{R}^{n_{x}}} I_{F}\left(\boldsymbol{x}_{I I I}, \boldsymbol{\theta}_{I I}^{*}\right) f_{\boldsymbol{X}}\left(\boldsymbol{x}_{I I I}, \boldsymbol{\vartheta}_{I I}^{*}\right) d \boldsymbol{x},
\end{aligned}
$$

to determine the lower bound of the probability of failure, and

$$
\begin{aligned}
\bar{P}_{f} & =P_{F}\left(\boldsymbol{\theta}_{I I}^{\bar{*}}, \boldsymbol{\vartheta}_{I I}^{\bar{*}}\right) \\
& =\int_{\boldsymbol{x} \in \mathbb{R}^{n_{x}}} I_{F}\left(\boldsymbol{x}_{I I I}, \boldsymbol{\theta}_{I I}^{\bar{*}}\right) f_{\boldsymbol{X}}\left(\boldsymbol{x}_{I I I}, \boldsymbol{\vartheta}_{I I}^{\bar{*}}\right) d \boldsymbol{z},
\end{aligned}
$$

to determine the upper bound. As such, the double loop is effectively broken since the propagation of epistemic and aleatory uncertainty is fully decoupled. The specific calculation of $\|\boldsymbol{D}\|_{p^{(1)}, p^{(2)}}$ is highly case dependent, as it depends both on the definition of $\boldsymbol{A}$, as on the types of uncertainty that are present in the model. The next sections will explore how several types of uncertainty, according to the classification provided in the introduction of this paper can be fitted into the operator norm framework. Finally, 
note that the integrals corresponding to $\bar{P}_{f}$ and $\underline{P}_{f}$ can be computed using any asymptotic approximation (FORM/SORM) or simulation method (Monte Carlo sampling, line sampling, directional importance sampling, SubSet simulation, etc.), depending on the problem at hand.

\subsection{Imprecisely defined zero-mean load with a deterministic model}

In case a deterministic model with imprecisely defined load is considered, the model that is presented in Eq. (5) can be rewritten as:

$$
y_{i}\left(\boldsymbol{\theta}_{I}, \boldsymbol{z}_{I V}\right)=\boldsymbol{A}_{i}\left(\boldsymbol{\theta}_{I}\right) \boldsymbol{z}\left(\boldsymbol{\xi}_{I I I}, \boldsymbol{\vartheta}_{I I}\right) .
$$

where $y_{i}$ denotes the $i^{\text {th }}$ response of the system and $\boldsymbol{A}_{i}$ the linear map of the input of the model to this response.

In case $\boldsymbol{z}\left(\xi_{I I I}, \boldsymbol{\vartheta}_{I I}\right)$ is a zero-mean stochastic load that is represented via the well-known Karhunen-Loève expansion (see Appendix A), this equation can be rewritten as:

$$
y_{i}\left(\boldsymbol{\theta}_{I}, \boldsymbol{z}_{I V}\right)=\boldsymbol{A}_{i}\left(\boldsymbol{\theta}_{I}\right) \boldsymbol{B}\left(\boldsymbol{\vartheta}_{I I}\right) \boldsymbol{\xi}_{I I I},
$$

where $\boldsymbol{\xi}_{I I I}$ is an $n_{K L}$-dimensional vector of i.i.d. standard normal random variables and $\boldsymbol{B}\left(\boldsymbol{\vartheta}_{\boldsymbol{I I}}\right) \in \mathbb{R}^{n_{z} \times n_{K L}}$ is a matrix collecting the basis functions of the Karhunen-Loève expansion that are obtained by solving the corresponding homogeneous Fredholm integral equation of the second kind. The form of this equation allows for a straightforward application of the operator norm framework, as also discussed in [45], by plugging it in into Eq. (25) as:

$$
\left\|\boldsymbol{A}_{i} \boldsymbol{B} \boldsymbol{\xi}\right\|_{p^{(1)}} \leq|c| \cdot\|\boldsymbol{\xi}\|_{p^{(2)}} .
$$

Hence, the operator norm for the $i^{\text {th }}$ response can in this case be computed as:

$$
\left\|\boldsymbol{A}_{i}\right\|_{p^{(1)}, p^{(2)}}=\sup \left\{\frac{\left\|\boldsymbol{A}_{i} \boldsymbol{B} \boldsymbol{\xi}\right\|_{p^{(1)}}}{\|\boldsymbol{\xi}\|_{p^{(2)}}}: \boldsymbol{\xi} \in \mathbb{R}^{n_{\xi}} \text { with } \boldsymbol{\xi} \neq 0\right\} .
$$

For the case of multiple responses $\boldsymbol{y}=y_{i}, i=1, \ldots, n_{y}$ the computation only changes slightly. Indeed, in this case a linear map $\boldsymbol{A}_{i}\left(\boldsymbol{\theta}_{I}\right) \boldsymbol{B}\left(\boldsymbol{\vartheta}_{I I}\right): \mathbb{R}^{n_{\xi}} \mapsto \mathbb{R}$, with its corresponding operator norm $\left\|\boldsymbol{A}_{i}\left(\boldsymbol{\theta}_{I}\right) \boldsymbol{B}\left(\boldsymbol{\vartheta}_{I I}\right)\right\|_{p^{(1)}, p^{(2)}}$ has to be considered for each of the $n_{y}$ responses of interest. In this case, a composite operator norm $\left\|\tilde{\boldsymbol{A}}\left(\boldsymbol{\theta}_{I}, \boldsymbol{\vartheta}_{I I}\right)\right\|_{p^{(1)}, p^{(2)}}$ has to be constructed to consider the joint effect of $\boldsymbol{\theta}_{I}$ and $\boldsymbol{\vartheta}_{I I}$ on all responses $y_{i}, i=1, \ldots, n_{y}$. The construction of the composite operator norm depends on the definition of how the different model responses contribute to the structure as being 'failed'. For instance, in the simplest case when for all responses of interest the failure domain corresponds to their respective maxima in case 
they are all bounded from above, $\left\|\tilde{\boldsymbol{A}}\left(\boldsymbol{\theta}_{I}, \boldsymbol{\vartheta}_{I I}\right)\right\|_{p^{(1)}, p^{(2)}}$ can be computed as:

$$
\left\|\tilde{\boldsymbol{A}}\left(\boldsymbol{\theta}_{I}, \boldsymbol{\vartheta}_{I I}\right)\right\|_{p^{(1)}, p^{(2)}}=\max _{i}\left\|\boldsymbol{A}_{i}\left(\boldsymbol{\theta}_{I}\right) \boldsymbol{B}\left(\boldsymbol{\vartheta}_{I I}\right)\right\|_{p^{(1)}, p^{(2)}}
$$

\subsection{Imprecisely defined load with a deterministic model}

The implicit assumption of a zero-mean load precludes the application of this framework to many realistic engineering cases such as e.g., wind loads on a building or mechanical loads on machinery parts. Evidently, when such load can be decomposed into a deterministic mean value and a stochastic variation around this mean, the application of the operator norm again becomes trivial. The real challenge is to account for loads with a non-zero mean component $\boldsymbol{z}_{\mathbf{0}}$ that may non-linearly depend on some type-II uncertain parameters $\boldsymbol{\vartheta}^{z_{0}}$. In this case, Eq. (23) has to be rewritten as:

$$
y_{i}\left(\boldsymbol{\theta}_{I}, \boldsymbol{z}_{I V}\right)=\boldsymbol{A}_{i}\left(\boldsymbol{\theta}_{I}\right)\left(\boldsymbol{z}_{\mathbf{0}}\left(\boldsymbol{\vartheta}_{I I}^{z_{0}}\right)+\boldsymbol{z}\left(\boldsymbol{\xi}_{I I I}, \boldsymbol{\vartheta}_{I I}\right)\right)
$$

which, taking the Karhunen-Loève series expansion of the stochastic part into account, becomes:

$$
y_{i}\left(\boldsymbol{\theta}_{I}, \boldsymbol{z}_{I V}\right)=\boldsymbol{A}_{i}\left(\boldsymbol{\theta}_{I}\right) \boldsymbol{z}_{\mathbf{0}}\left(\boldsymbol{\vartheta}_{I I}^{z_{0}}\right)+\boldsymbol{A}_{i}\left(\boldsymbol{\theta}_{I}\right) \boldsymbol{B}\left(\boldsymbol{\vartheta}_{I I}\right) \boldsymbol{\xi}_{I I I} .
$$

It is clear that this equation no longer fits the required form to apply the operator norm framework, as prescribed in Eq. (16). However, since the equation still represents an affine transformation from $\boldsymbol{\xi}$ to $y_{i}$, it can be rephrased via augmented matrix formulation as:

$$
\left[\begin{array}{c}
y_{i}\left(\boldsymbol{\theta}_{I}, \boldsymbol{z}_{I V}\right) \\
1
\end{array}\right]=\boldsymbol{T}_{i}\left(\boldsymbol{\theta}_{I}, \boldsymbol{\vartheta}_{I I}^{z_{0}}, \boldsymbol{\vartheta}_{I I}\right)\left[\begin{array}{c}
\boldsymbol{\xi}_{I I I} \\
1
\end{array}\right]
$$

with $\boldsymbol{T}_{i}\left(\boldsymbol{\theta}_{I}, \boldsymbol{\vartheta}_{I I}^{z_{0}}, \boldsymbol{\vartheta}_{I I}\right) \in \mathbb{R}^{\left(n_{y}+1\right) \times\left(n_{\xi}+1\right)}$ a block-matrix that is defined as:

$$
\boldsymbol{T}_{i}\left(\boldsymbol{\theta}_{I}, \boldsymbol{\vartheta}_{I I}^{z_{0}}, \boldsymbol{\vartheta}_{I I}\right)=\left[\begin{array}{cc}
{\left[\boldsymbol{A}_{i}\left(\boldsymbol{\theta}_{I}\right) \boldsymbol{B}\left(\boldsymbol{\vartheta}_{I I}\right)\right]} & {\left[\boldsymbol{A}_{i}\left(\boldsymbol{\theta}_{I}\right) \boldsymbol{z}_{\mathbf{0}}\left(\boldsymbol{\vartheta}_{I I}^{z_{0}}\right)\right]} \\
\mathbf{0} & 1
\end{array}\right]
$$

It is straightforward to prove that this formulation is completely equivalent to the original formulation 
given in Eq. (16). As such, the operator norm can in this case be computed as:

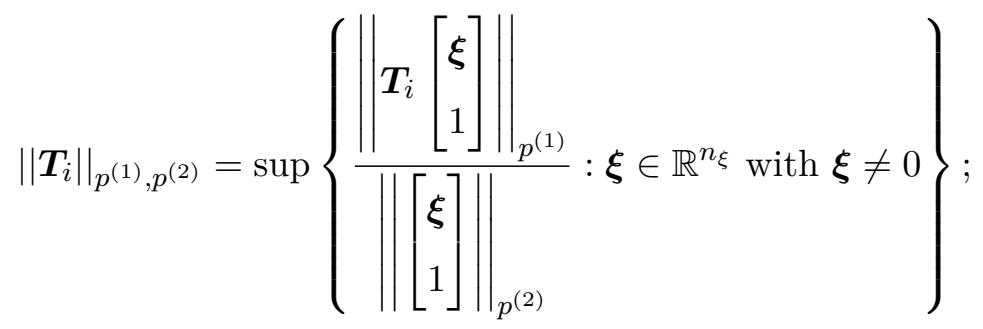

the extension of this equation to multiple responses is analogous to Eq. (27) and will not be discussed in detail.

\subsection{Imprecisely defined load with a type-II uncertain model}

In case the model, represented by the linear map $\boldsymbol{A}_{i}$ becomes subjected to type-II uncertainty as well, a response of the structure to a non-zero mean load can be described as:

$$
y_{i}\left(\boldsymbol{\theta}_{I I}, \boldsymbol{z}_{I V}\right)=\boldsymbol{A}_{i}\left(\boldsymbol{\theta}_{I I}\right) \boldsymbol{z}_{\mathbf{0}}\left(\boldsymbol{\vartheta}_{I I}^{z_{0}}\right)+\boldsymbol{A}_{i}\left(\boldsymbol{\theta}_{I I}\right) \boldsymbol{B}\left(\boldsymbol{\vartheta}_{I I}\right) \boldsymbol{\xi}_{I I I}
$$

This corresponds for instance to a continuum problem where a structure is subjected to an imprecise stochastic load (e.g., a wind load with an imprecisely defined spectrum), but where the analyst furthermore has insufficient data to make crisp decisions on the actual parameters of the structure, such as e.g., Young's modulus of applied materials. In general, this allows the analyst to jointly take epistemic uncertainty in the model definition as well as in the stochastic load definition into account. This statement holds as long as the linear map (e.g., representing the inverse of the stiffness matrix in a linear elastic case) has an explicit dependence on the epistemically uncertain model parameters under consideration.

In this case, the augmented matrix $\boldsymbol{T}_{i}\left(\boldsymbol{\theta}_{I I}, \boldsymbol{\vartheta}_{I I}^{z_{0}}, \boldsymbol{\vartheta}_{I I}\right) \in \mathbb{R}^{\left(n_{y}+1\right) \times\left(n_{\xi}+1\right)}$ can be described as:

$$
\boldsymbol{T}_{i}\left(\boldsymbol{\theta}_{I I}, \boldsymbol{\vartheta}_{I I}^{z_{0}}, \boldsymbol{\vartheta}_{I I}\right)=\left[\begin{array}{cc}
{\left[\boldsymbol{A}_{i}\left(\boldsymbol{\theta}_{I I}\right) \boldsymbol{B}\left(\boldsymbol{\vartheta}_{I I}\right)\right]} & {\left[\boldsymbol{A}_{i}\left(\boldsymbol{\theta}_{I I}\right) \boldsymbol{z}_{\mathbf{0}}\left(\boldsymbol{\vartheta}_{I I}^{z_{0}}\right)\right]} \\
\mathbf{0} & 1
\end{array}\right]
$$

with the corresponding operator norm given by Eq. (32). It is important to note that in general, it is not possible to account for Type-III uncertainties in the model definition, as such formulation does not allow for rephrasing the model in the prescribed format to apply the operator norm theory (see Eq. (16)).

\subsection{Practical computation of the operator norms}

The definitions of the operator norm, as e.g., given in Eq. (32) is not possible without defining the norms on both sides of Eq. (10). In real life applications, this selection should be made with care since the applicability of the method depends on it. In the following we give some pointers on how this selection 
should be made. In essence, the selection of the value of $p^{(1)}$ is inherently connected to the definition of the reliability problem under consideration. For instance, if failure is described as one of the responses exceeding a prescribed threshold within a certain time interval, which corresponds to the first-excursion probability, then the infinity norm such that $p^{(1)} \rightarrow \infty$ is a suitable selection, as illustrated in earlier work of the authors in [45] and [49]. Regarding $p^{(2)}$, numerical experience suggest that it should be selected such that $p^{(2)}=2$, as this can be loosely interpreted as the energy content associated with the external loading. Based on the information in Table 1 , taking $p^{(1)} \rightarrow \infty$ and $p^{(2)}=2$ for the case of calculating the probability of failure, the operator norm corresponds to the largest $\mathcal{L}_{2}$ norm of a row of the matrix representing the linear map. In the zero-mean load case with a deterministic linear map, the operator norm is as such computed as:

$$
\left\|\boldsymbol{D}\left(\boldsymbol{\theta}_{I}, \boldsymbol{\vartheta}_{\boldsymbol{I I}}\right)\right\|_{p^{(1)}, p^{(2)}}=\max _{k=1, \ldots, n_{y}}\left\|\boldsymbol{M}_{i, k:}\left(\boldsymbol{\theta}_{I}, \boldsymbol{\vartheta}_{\boldsymbol{I I}}\right)\right\|_{2}
$$

with $\boldsymbol{M}_{i}\left(\boldsymbol{\theta}_{I}, \boldsymbol{\vartheta}_{\boldsymbol{I I}}\right)=\boldsymbol{A}_{i}\left(\boldsymbol{\theta}_{I}\right) \boldsymbol{B}\left(\boldsymbol{\vartheta}_{I I}\right)$, and where the subscript $k$ : denotes taking the $k^{\text {th }}$ row of the matrix $\boldsymbol{M}_{i}\left(\boldsymbol{\theta}_{I}, \boldsymbol{\vartheta}_{\boldsymbol{I I}}\right)$. Similarly, the operator norm for case where a non-zero mean load is considered can be computed as:

$$
\left\|\boldsymbol{D}\left(\boldsymbol{\theta}_{I}, \boldsymbol{\vartheta}_{I I}^{z_{0}}, \boldsymbol{\vartheta}_{\boldsymbol{I I}}\right)\right\|_{p^{(1)}, p^{(2)}}=\max _{k=1, \ldots, n_{y}}\left\|\boldsymbol{T}_{i, k:}\left(\boldsymbol{\theta}_{I}, \boldsymbol{\vartheta}_{I I}^{z_{0}}, \boldsymbol{\vartheta}_{\boldsymbol{I I}}\right)\right\|_{2}
$$

Note that the maximum is taken over the first $n_{y}$ rows of $\boldsymbol{T}_{i, k:}\left(\boldsymbol{\theta}_{I}, \boldsymbol{\vartheta}_{I I}^{z_{0}}, \boldsymbol{\vartheta}_{\boldsymbol{I I}}\right)$. This is reasonable, since the last row of the matrix is related with the value 1 in the left-hand side of the equation, which is an artefact from the reformulation in the augmented matrix form. Finally, operator norm for the general load case in combination with a linear map that is subjected to epistemic uncertainty can be computed as:

$$
\left\|\boldsymbol{D}\left(\boldsymbol{\theta}_{I I}, \boldsymbol{\vartheta}_{I I}^{z_{0}}, \boldsymbol{\vartheta}_{\boldsymbol{I I}}\right)\right\|_{p^{(1)}, p^{(2)}}=\max _{k=1, \ldots, n_{y}}\left\|\boldsymbol{T}_{i, k:}\left(\boldsymbol{\theta}_{I I}, \boldsymbol{\vartheta}_{I I}^{z_{0}}, \boldsymbol{\vartheta}_{\boldsymbol{I I}}\right)\right\|_{2}
$$

Those parameters of the imprecise stochastic input to the model can then in the most general case be determined by solving the following constrained optimization problems:

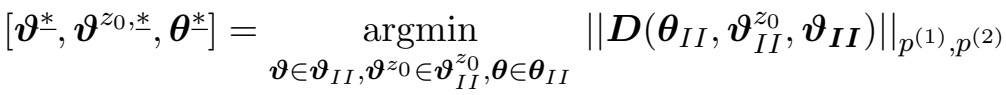

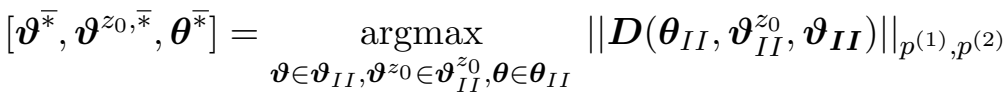

To solve these optimization problems, any numerical optimizer can be used, depending on the convexity and smoothness of the functional relation between $\|\boldsymbol{D}\|_{p^{(1)}, p^{(2)}}$ and $\left[\boldsymbol{\vartheta}, \boldsymbol{\vartheta}^{z_{0}}, \boldsymbol{\theta}\right]$. Concerning the computational cost of performing these optimization problems, following remarks should be made:

- In case the model is deterministic (i.e., considering $\boldsymbol{\theta}_{I}$ ), repeated evaluations of Eq. (38) and Eq. (39) 
for several values of $\boldsymbol{\vartheta} \in \boldsymbol{\vartheta}_{I I}$ and $\boldsymbol{\vartheta}^{z_{0}} \in \boldsymbol{\vartheta}_{I I}^{z_{0}}$ do not require a re-evaluation of $\boldsymbol{A}_{i}$, which is convenient from a numerical standpoint in case this map is represented by e.g., a finite element model. It should furthermore be noted that in case the load becomes interval-valued (i.e., $\boldsymbol{\vartheta}, \boldsymbol{\vartheta}^{z_{0}}$ and/or $\boldsymbol{\xi}$ become interval valued), this problem reduces to a regular propagation of intervals on the structure. In this case, the presented approach reduces to the well-known anti-optimisation framework for propagating intervals [4].

- In case the model is subjected to epistemic uncertainty, a re-evaluation of $\boldsymbol{A}_{i}$ for each realisation of $\boldsymbol{\theta} \in \boldsymbol{\theta}^{\boldsymbol{I I}}$ is required to assess the effect of $\boldsymbol{\theta}$ on the operator norm. More specifically, at each step of the optimiser, the linear map $\boldsymbol{A}_{i}$ has to be constructed. For example, in the case of static finite element model, the computation of $\boldsymbol{A}_{i}$ corresponds to assembling the stiffness matrix of the model and taking its inverse. Alternatively, in case dynamic time domain calculations are performed, the impulse response functions of the model have to be computed, as e.g., discussed in [45]. Since this can entail a non-negligible calculation cost, this step of the procedure might consume a considerable amount of computational power. Nonetheless, it is still orders of magnitude more efficient than having to solve a reliability problem at each step of the optimisation (as performed in a classical double-loop approach).

\section{Numerical examples}

\subsection{Example 1: a fully clamped plate}

\subsubsection{Model introduction and uncertainty definition}

The first example deals with a model of a thin steel plate of $1[\mathrm{~m}]$ by $1[\mathrm{~m}]$ that is fully clamped at one side. The plate is subjected to a distributed load over the top surface, and its displacement $\boldsymbol{u}$ is computed using a finite element model consisting of 100 evenly distributed linear shell elements, resulting in 121 nodes. As such, there are 110 active nodes in the model. In the analysis, the degrees of freedom per node correspond to one translation and two rotations. The distributed load is modelled as the sum of a mean component with a zero-mean isotropic two-dimensional Gaussian random field that is governed by a squared exponential covariance kernel, and is modeled as:

$$
F\left(\boldsymbol{r}, \boldsymbol{\vartheta}_{I I}, \boldsymbol{\xi}\right)=\mathbf{1} \vartheta_{1} * \sin \left(\pi / \vartheta_{2}\right)+\vartheta_{3} \boldsymbol{B}\left(\vartheta_{4}, \boldsymbol{r}\right) \boldsymbol{\xi}
$$

with $\vartheta=\left[\vartheta_{1}^{I}, \vartheta_{2}^{I}, \vartheta_{3}^{I}, \vartheta_{4}^{I}\right]$ the set of epistemic uncertainties, $\boldsymbol{r} \in \Omega \subset \mathbb{R}^{2}$ a spatial coordinate inside the model geometry $\Omega=[0,1]^{2} \mathrm{~m}, \mathbf{1} \in \mathbb{R}^{110}$ a vector whose components are equal to $1, \boldsymbol{B}\left(\vartheta_{4}, \boldsymbol{r}\right) \in \mathbb{R}^{100 \times 10}$ the basis of the random field obtained via the Karhunen-Loève expansion retaining the first 10 eigenmodes and $\boldsymbol{\xi}$ a vector containing 10 standard normal random variables (see also Appendix A). Physically speaking, $\vartheta_{3}$ 
represents the standard deviation of the random field load and $\vartheta_{4}$ the correlation length. The corresponding equilibrium equation associated with the finite element model of the plate is represented as:

$$
\boldsymbol{K}(\boldsymbol{\theta}) \boldsymbol{u}=\boldsymbol{G}\left(\mathbf{1} \vartheta_{1} * \sin \left(\pi / \vartheta_{2}\right)+\vartheta_{3} \boldsymbol{B}\left(\vartheta_{4}, \boldsymbol{r}\right) \boldsymbol{\xi}\right),
$$

with $\boldsymbol{K} \in \mathbb{R}^{330 \times 330}$ the stiffness matrix of the plate; $\boldsymbol{\theta}=[E, t]$, with $E$ representing Young's modulus and $t$ the thickness of the plate; $\boldsymbol{G}$ is a matrix that couples the loading with the degrees-of-freedom of the finite element model; and $\boldsymbol{u} \in \mathbb{R}^{330}$ the resulting displacement vector. It is assumed that the degrees-of-freedom of the finite element model have beer ordered such that the first 110 components of $\boldsymbol{u}$ correspond to vertical displacements. The linear map that has to be used to compute the operator norm is as such given as:

$$
\boldsymbol{T}_{i}(\boldsymbol{\theta}, \boldsymbol{\vartheta})=\left[\begin{array}{cc}
{\left[\boldsymbol{K}^{-1}(\boldsymbol{\theta}) \boldsymbol{G} \vartheta_{3} \boldsymbol{B}\left(\vartheta_{4}, \boldsymbol{r}\right)\right]} & {\left[\boldsymbol{K}^{-1}(\boldsymbol{\theta}) \boldsymbol{G}\left(\mathbf{1} \vartheta_{1} * \sin \left(\pi / \vartheta_{2}\right)\right)\right]} \\
\mathbf{0} & 1
\end{array}\right]
$$

and the corresponding augmented matrix is computed similarly to Eq. (34). Failure of the plate is in this case study defined as the situation where the displacement $u_{i}$ of the left free corner node of the plate exceeds a threshold, specifically $\left|u_{i}\right|>0.15[\mathrm{~m}], i=110$. Hence, the operator norm is computed with $p^{(1)} \rightarrow \infty$ and $p^{(2)}=2$. In this case study, following intervals are considered: $\vartheta_{1}=[7.5 ; 12.5][\mathrm{N}], \vartheta_{2}=[1 ; 5], \vartheta_{3}=[0.5 ; 1.5]$ $[\mathrm{N}], \vartheta_{4}=[0.5 ; 3.0][\mathrm{m}], E=[1.85 ; 2.25] \cdot 10^{+11}[\mathrm{~Pa}]$ and $t=[4.8 ; 5.2][\mathrm{mm}]$.

Those parameters that yield the bounds of the probability of failure are determined by solving the following optimization problems:

$$
\boldsymbol{\theta}^{*}, \boldsymbol{\vartheta}^{*}=\underset{\boldsymbol{\theta} \in \boldsymbol{\theta}^{I}, \boldsymbol{\vartheta} \in \boldsymbol{\vartheta}^{I}}{\operatorname{argmin}} \max _{k=1, \ldots, n_{y}}\left\|\boldsymbol{T}_{i, k:}\left(\boldsymbol{\theta}_{I I}, \boldsymbol{\vartheta}_{\boldsymbol{I I}}\right)\right\|_{2}
$$

to determine those parameters that yield the lower bound and:

$$
\boldsymbol{\theta}^{\bar{*}}, \boldsymbol{\vartheta}^{\bar{*}}=\underset{\boldsymbol{\theta} \in \boldsymbol{\theta}^{I}, \boldsymbol{\vartheta} \in \boldsymbol{\vartheta}^{I}}{\operatorname{argmax}} \max _{k=1, \ldots, n_{y}}\left\|\boldsymbol{T}_{i, k:}\left(\boldsymbol{\theta}_{I I}, \boldsymbol{\vartheta}_{\boldsymbol{I I}}\right)\right\|_{2}
$$

to determine those parameters that yield the upper bound, with $\boldsymbol{\theta}=[E, t]$.

\subsubsection{Univariate uncertainty propagation}

The effect of varying each epistemically uncertain parameter separately on the operator norm $\|\boldsymbol{D}\|_{p^{(1)}, p^{(2)}}$ as well as on the probability of failure $P_{f}$ of the plate is shown in Figure 1. These plots are obtained by drawing 100 Latin Hypercube samples in between the bounds of the intervals on the 6 epistemic parameters under the assumption of a uniform distribution, and computing the corresponding operator norm and failure probability. Note that this uniform distribution is solely applied to visualize the relationship between these 


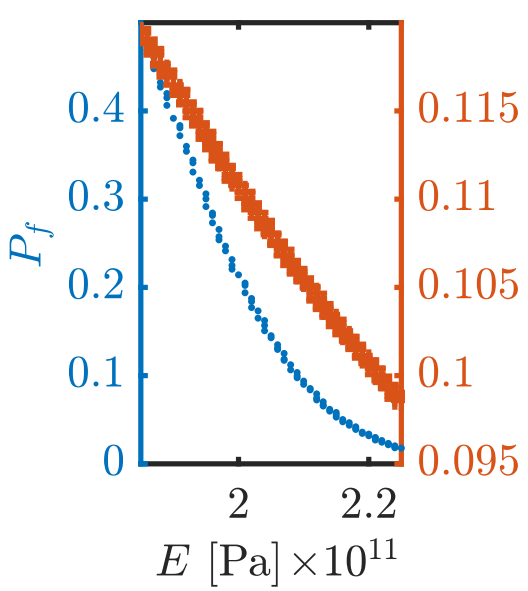

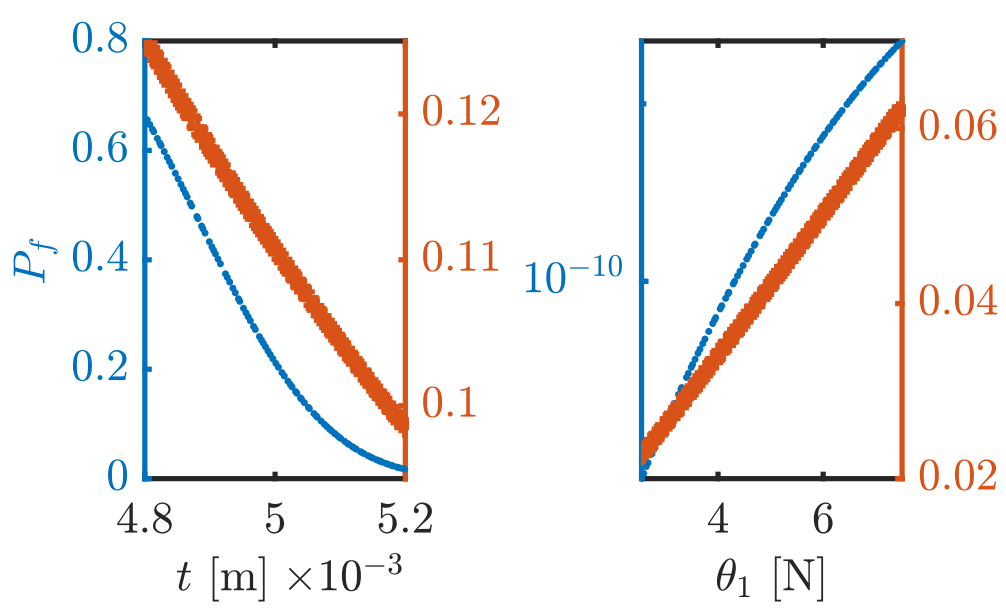

parameters, $\|\boldsymbol{D}\|_{p^{(1)}, p^{(2)}}$ and $P_{f}$. The operator norm is computed using Eq. (37), whereas the probability of failure is computed using FORM. Since the limit state function is linear with respect to $\boldsymbol{\xi}$ and the stochastic dimension of the problem is low, this is a reasonable choice that furthermore allows a rigorous study of the problem within reasonable computational cost. From this figure, it is clear that a perfect correlation exists between the extrema in $\|\boldsymbol{D}\|_{p^{(1)}, p^{(2)}}$ and $P_{f}$. However, to compute $\|\boldsymbol{D}\|_{p^{(1)}, p^{(2)}}$, no propagation of the aleatory uncertainty through the model is required. This illustrates that in a univariate case, those values for the epistemic parameters that yield the extrema in $P_{f}$ can be determined without having to solve the probability integrals at each step of the optimization, as is the case in Eq. (6) and Eq. (8). For the tested parameters, this relationship is furthermore smooth, enabling the use of highly-efficient gradient based optimization algorithms such as Quasi-Newton approaches.
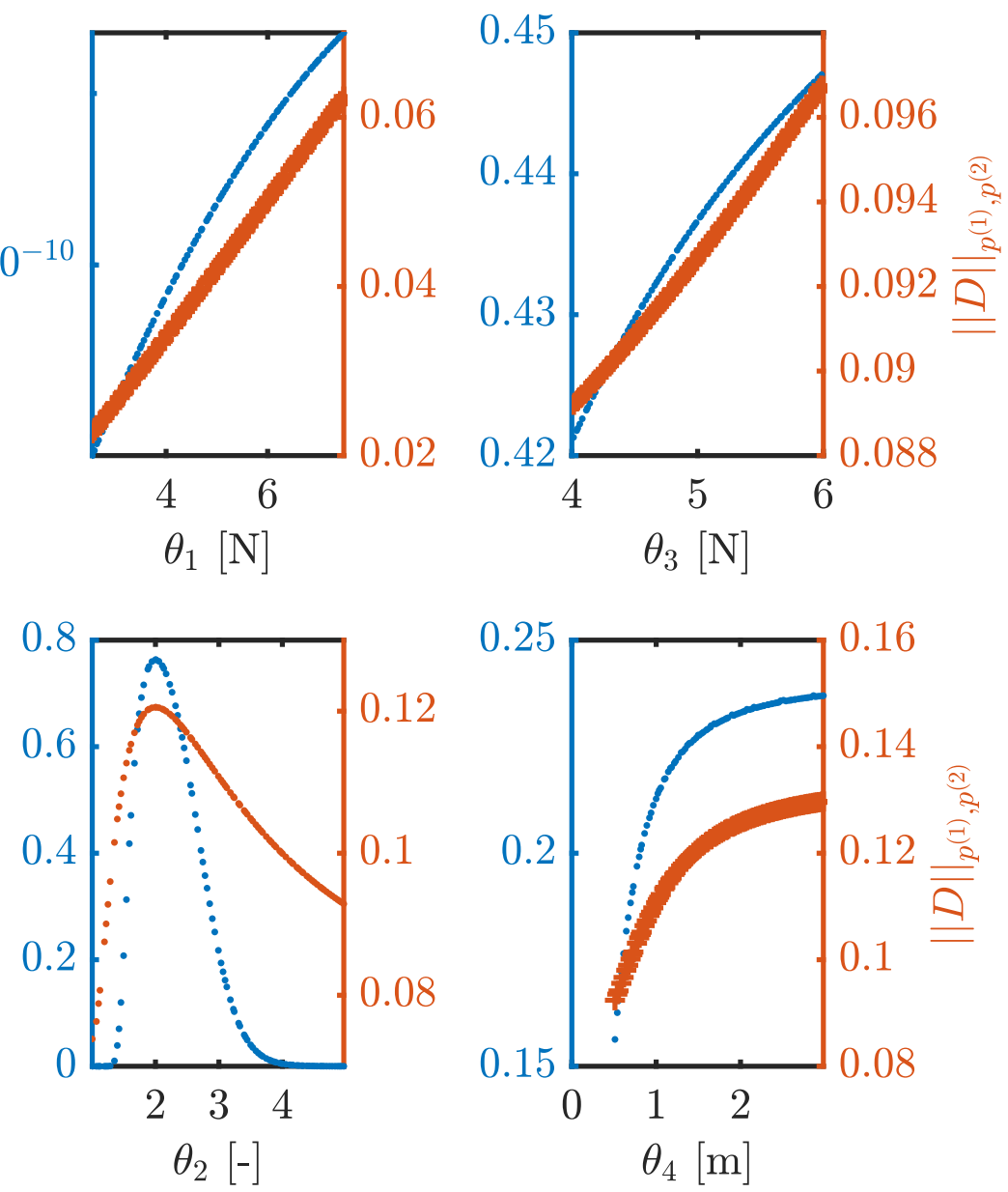

Figure 1: Effect of varying each parameter separately on the operator norm $\|\boldsymbol{D}\|_{p^{(1)}, p^{(2)}}$ (orange plus signs) as well as on the probability of failure $P_{f}$ (blue dots) 


\subsubsection{Joint uncertainty propagation}

To solve this case using operator norm theory, the optimization problems defined in Eq. (43) and Eq. (44) are solved using Particle Swarm Optimization (PSO) [50]. Afterwards, based on the result of these optimizations, the corresponding probabilities of failure are computed using FORM. The main computational cost in this approach lies in the repeated evaluation of the inverse of the stiffness matrix of the plate to compute $\|\boldsymbol{D}\|_{p^{(1)}, p^{(2)}}$ at each iteration of the optimizer.

To verify the accuracy of the obtained results, as well as to highlight the effectiveness and efficiency of the operator norm approach, the results obtained via this method are compared to two other commonly applied approaches to solve this type of problems:

Vertex analysis: The outer optimization loop, as introduced in Eq. (6) and Eq. (8) is replaced by a combinatorial search of the vertices of the epistemic hyper-cube defined by $\boldsymbol{\vartheta}^{I} \times E^{I} \times t^{I}$, where $\times$ denotes the Cartesian product. FORM is used to compute the probability of failure for each vertex.

Solving the double loop directly: The propagation is performed by solving Eq. (6) and Eq. (8) directly, where for each step of the Particle Swarm Optimization in the outer loop, a FORM estimate of $P_{f}$ is performed.

The main computational cost in these approaches lies in the repeated solution of the probability integral to estimate $P_{f}$ for each vertex. Specifically, this calculation has to be performed for each particle position in the particle swarm optimization procedure. All computations are performed on a server equipped with 512 GB of RAM and a 64-core AMD EPYC $6701 \mathrm{CPU} @ 2.65 \mathrm{GHz}$. A single evaluation of this FE model requires approx. $15[\mathrm{~s}]$ on this machine (including computational overhead).

The results of these computations are illustrated in Table 2; also the operator norms corresponding to the bounds corresponding to the optimum that is obtained by the Vertex Analysis and Double loop approach are listed for the sake of comparison. As can be noted, the results obtained by the optimization over the Operator Norm and the double loop match up to the numerical precision of the Particle Swarm Optimizer. This shows that also in case all parameters are jointly considered, the decoupled propagation using the operator norm is capable of predicting the correct bounds on the probability of failure. It is important to note that this estimate comes at a greatly reduced computational cost, since no repeated solution for $P_{f}$ is required, as is evidenced in the Table. Indeed, the computation of the upper bound for instance using the operator norm required 880 model evaluations to determine $\boldsymbol{\vartheta}^{\bar{*}}+$ an additional 33 evaluations to compute $P_{f}$ using FORM. The double loop approach on the other hand required 22957 model evaluations to derive the same estimate. It should furthermore be noted that this difference will be further amplified in case simulation methods are used to determine $P_{f}$. This Table also shows that the Vertex method, although in 
this case also highly efficient since both bounds are obtained in the same propagation loop, fails to provide the correct bounds, which is caused by the non-monotonous relationship between $P_{f}$ and $\vartheta_{2}$.

Table 2: Results obtained by (1) applying the Vertex analysis, (2) optimising over the operator norm and (3) solving the double loop problem on the case with an epistemically uncertain linear map

\begin{tabular}{|l|cc|cc|cc|}
\hline & \multicolumn{2}{|c|}{ Vertex analysis } & \multicolumn{2}{c|}{$\min || \boldsymbol{D} \|_{p^{(1)}, p^{(2)}}$} & \multicolumn{2}{c|}{$\min P_{f}$} \\
& $\boldsymbol{\vartheta}^{*}$ & $\boldsymbol{\vartheta}^{\text {* }}$ & $\boldsymbol{\vartheta}^{*}$ & $\boldsymbol{\vartheta}^{\text {* }}$ & $\boldsymbol{\vartheta}^{*}$ & $\boldsymbol{\vartheta}^{\text {* }}$ \\
\hline$\|\boldsymbol{D}\|_{p^{(1)}, p^{(2)}}$ & 0.0208 & 0.0859 & 0.0208 & 0.1112 & 0.0208 & 0.1112 \\
$P_{f}$ & $8.67 \cdot 10^{-06}$ & 0.2907 & $8.67 \cdot 10^{-06}$ & 0.4889 & $8.67 \cdot 10^{-06}$ & 0.4889 \\
\hline$n^{0}$ FE solutions & \multicolumn{2}{|c|}{1794} & $640+47$ & $880+33$ & 18156 & 26539 \\
\hline
\end{tabular}

\subsection{Example 2: building model}

The second example involves the six story reinforced concrete building model depicted in Figure 2, which is borrowed from [51]. Each floor plan is of square shape with side length $32 \mathrm{~m}$ and story height of 3.6 [m]. All floor slabs possess a thickness of $20 \mathrm{~cm}$ and are supported by a C-shaped shear wall of 20 [cm] thickness and 16 columns of square cross section with side length $40[\mathrm{~cm}]$. The Young's modulus is set equal to $2.3 \times 10^{10}[\mathrm{~Pa}]$. It is assumed that the building undergoes small displacements and hence, it is modeled as linear elastic. The behavior of the building is characterized by means of a finite element model that comprises about 9500 shell and beam elements and more than $50 \times 10^{3}$ degrees-of-freedom. The building is excited by a stochastic ground acceleration along the $y$ direction. This ground acceleration is generated considering a modulated Clough-Penzien (CP) model (see Appendix B), with nominal parameters $\vartheta=$ $\left[\omega_{g}, \omega_{f}, \zeta_{g}, \zeta_{f}, S_{0}, c_{1}, c_{2}\right]=\left[4 \pi[\mathrm{rad} / \mathrm{s}], 0.4 \pi[\mathrm{rad} / \mathrm{s}], 0.7,0.7,3 \times 10^{-4}\left[\mathrm{~m}^{2} / \mathrm{s}^{3}\right], 0.14,0.16\right]$. The total duration of the acceleration is $20 \mathrm{~s}$ and the time step discretization is $\Delta t=0.01 \mathrm{~s}$. Due to design purposes, it is of interest to control that the interstory drifts along the $y$ direction do not exceed a threshold level of $2 \times 10^{-3}$ times the story height. These interstory drifts are controlled at five points, between nodes $n_{2}-n_{1}, n_{3}-n_{2}$, $n_{4}-n_{3}, n_{5}-n_{4}$ and $n_{6}-n_{5}$, as illustrated in Figure 2 . The probability of failure, represented by a first excursion probability, is computed using Directional Importance Sampling [17] with a sample size of 500 deterministic model evaluations. In this case study, 13 epistemically uncertain parameters are considered on top of this aleatory uncertain load: the 7 parameters corresponding to the modulated Clough-Penzien autocorrelation spectrum, as well as Young's modulus of each floor slab of the building. These values are represented in Table 3 .

The interstory drift values are computed using the convolution method explained in Appendix C. Via 


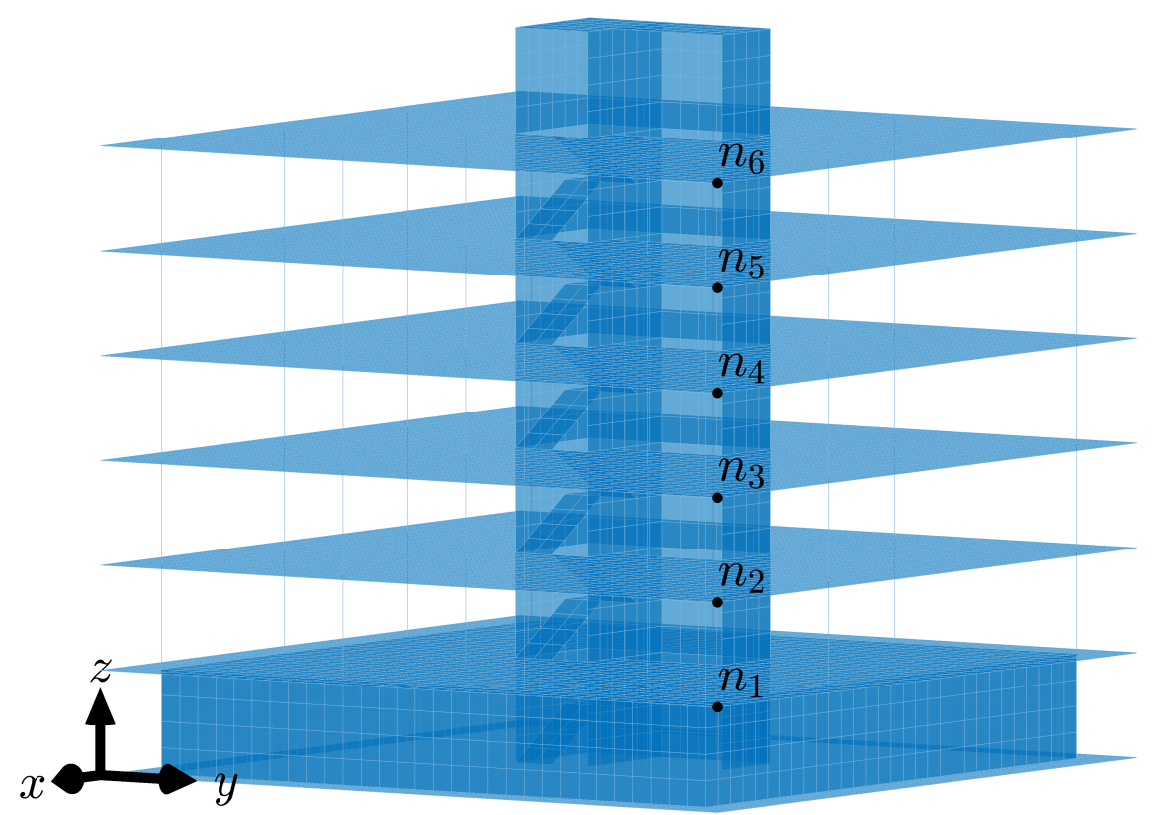

Figure 2: Example 2 - Isometric view of the building model

this approach, a linear map $\boldsymbol{A}$ is calculated per interstory drift as:

$$
\boldsymbol{A}_{i}=\left[\begin{array}{c}
\boldsymbol{a}_{i, 1}^{T} \\
\boldsymbol{a}_{i, 2}^{T} \\
\vdots \\
\boldsymbol{a}_{i, 2001}^{T}
\end{array}\right]
$$

423 with $i=1,2, \ldots, 5$. Then, based on this set of 5 maps, the following optimization problems are solved to

Table 3: Bounds of the epistemic parameters in the building model

\begin{tabular}{lll}
\hline & Lower bound & Upper bound \\
\hline$\omega_{g}^{I}$ & $2.40 \pi[\mathrm{rad} / \mathrm{s}]$ & $8 \pi[\mathrm{rad} / \mathrm{s}]$ \\
$\omega_{f}^{I}$ & $0.24 \pi[\mathrm{rad} / \mathrm{s}]$ & $0.8 \pi[\mathrm{rad} / \mathrm{s}]$ \\
$\zeta_{g}^{I}$ & 0.6 & 0.85 \\
$\zeta_{f}^{I}$ & 0.6 & 0.85 \\
$S_{0}^{I}$ & $2.25 \times 10^{-4}\left[\mathrm{~m}^{2} / \mathrm{s}^{3}\right]$ & $3.75 \times 10^{-4}\left[\mathrm{~m}^{2} / \mathrm{s}^{3}\right]$ \\
$c_{1}^{I}$ & 0.12 & 0.16 \\
$c_{2}^{I}$ & 0.14 & 0.18 \\
$E$ & $2.07 \times 10^{+10}[\mathrm{~Pa}]$ & $2.53 \times 10^{+10}[\mathrm{~Pa}]$ \\
\hline
\end{tabular}


determine those epistemic parameters that provide the bounds on the probability of failure:

$$
\left[\boldsymbol{E}^{*}, \boldsymbol{\vartheta}^{*}\right]=\underset{\boldsymbol{E} \in \boldsymbol{E}^{I}, \boldsymbol{\vartheta} \in \boldsymbol{\vartheta}^{I}}{\operatorname{argmin}} \max _{i} \max _{l} \boldsymbol{A}_{i, l}
$$

to determine those parameters that yield the lower bound and:

$$
\left[\boldsymbol{E}^{\bar{*}}, \boldsymbol{\vartheta}^{\bar{*}}\right]=\underset{\boldsymbol{E} \in \boldsymbol{E}^{I}, \boldsymbol{\vartheta} \in \boldsymbol{\vartheta}^{I}}{\operatorname{argmax}} \max _{i} \max _{l} \boldsymbol{A}_{i, l:}
$$

to determine those parameters that yield the upper bound. These optimization problems are solved using Particle Swarm Optimization. The corresponding probability of failure values are subsequently computed via Directional Importance Sampling.

Also in this case study, the presented approach based on operator norm theory is compared against two other commonly applied approaches to illustrate the effectiveness and efficiency. Specifically, it is compared against:

- a vertex analysis, where all combinations of the bounds of the parameters in $\boldsymbol{\vartheta}^{I}$ are combined, leading to $2^{13}=8192$ computations of the probability of failure and hence, 4096000 deterministic model evaluations

- Quasi Monte Carlo simulation under the assumption of a uniform distribution between the bounds in $\boldsymbol{\vartheta}^{I}$ comprising of a Sobol sequence with 10000 points, leading to 10000 computations of the probability of failure and hence, 5000000 deterministic model evaluations,

The results of the propagation of the uncertainty through the building model, obtained by performing the three propagation approaches as discussed above are illustrated in Table 4 . In this table, $\tilde{\boldsymbol{D}}=$ $\max _{i} \max _{l} \boldsymbol{A}_{i, l:}$. From this table, it is clear that the Operator norm optimization is perfectly capable of bounding the probability of failure on the structure, given the sources of uncertainty, at greatly reduced computational cost in comparison to the other approaches. In fact, the upper bound of $P_{f}$ is not perfectly captured by the Vertex method, which can be explained by a possible non-monotonic relationship between the parameters and input of the model and $P_{f}$, which is caused interplay between the frequency content of the non-stationary stochastic base excitation with resonances inside the structure. Since the Vertex method requires such monotonicity, it underestimates the upper bound. Finally, it is shown that applying Quasi Monte Carlo simulation to replace the outer loop in this case gives a large under-estimation of the bounds on $P_{f}$, despite the extremely high computational cost.

These conclusions are furthermore confirmed by Figure 3. This figure shows $P_{f}$ plotted against $\|\tilde{\boldsymbol{D}}\|_{p^{(1)}, p^{(2)}}$, where $P_{f}$ is obtained by performing a Vertex analysis (blue crosses), Quasi Monte Carlo sampling (orange 
Table 4: Results obtained by (1) applying the Vertex analysis, (2) replacing the outer loop with Quasi Monte Carlo and (3) optimizing over the operator norm

\begin{tabular}{|ll|l|l|l|}
\hline & & $\|\tilde{D}\|_{p^{(1)}, p^{(2)}}$ & $P_{f}$ & $\mathrm{n}^{0}$ FE solutions \\
\hline \multirow{2}{*}{ Vertex analysis } & $\boldsymbol{\vartheta}^{*}$ & 0.0012 & $6.328 \cdot 10^{-08}$ & \multirow{2}{*}{4096000} \\
& $\boldsymbol{\vartheta}^{\bar{*}}$ & 0.0025 & 0.0855 & \\
\hline \multirow{2}{*}{ Quasi Monte Carlo } & $\boldsymbol{\vartheta}^{*}$ & 0.0013 & $9.0432 \cdot 10^{-07}$ & \multirow{2}{*}{5000000} \\
& $\boldsymbol{\vartheta}^{\bar{*}}$ & 0.0023 & 0.0481 & \\
\hline \multirow{2}{*}{$\min \|\boldsymbol{A}\|_{p^{(1)}, p^{(2)}}$} & $\boldsymbol{\vartheta}^{*}$ & 0.0012 & $6.59 \cdot 10^{-08}$ & $500+3000$ \\
& $\boldsymbol{\vartheta}^{\bar{*}}$ & 0.0025 & 0.0894 & $500+2100$ \\
\hline
\end{tabular}

dots) and the optimization approach based on operator norm theory that is introduced in this paper (black diamonds). This figure furthermore shows that a reasonably smooth and monotonically increasing relationship between $P_{f}$ and $\|\tilde{\boldsymbol{D}}\|_{p^{(1)}, p^{(2)}}$ exists.

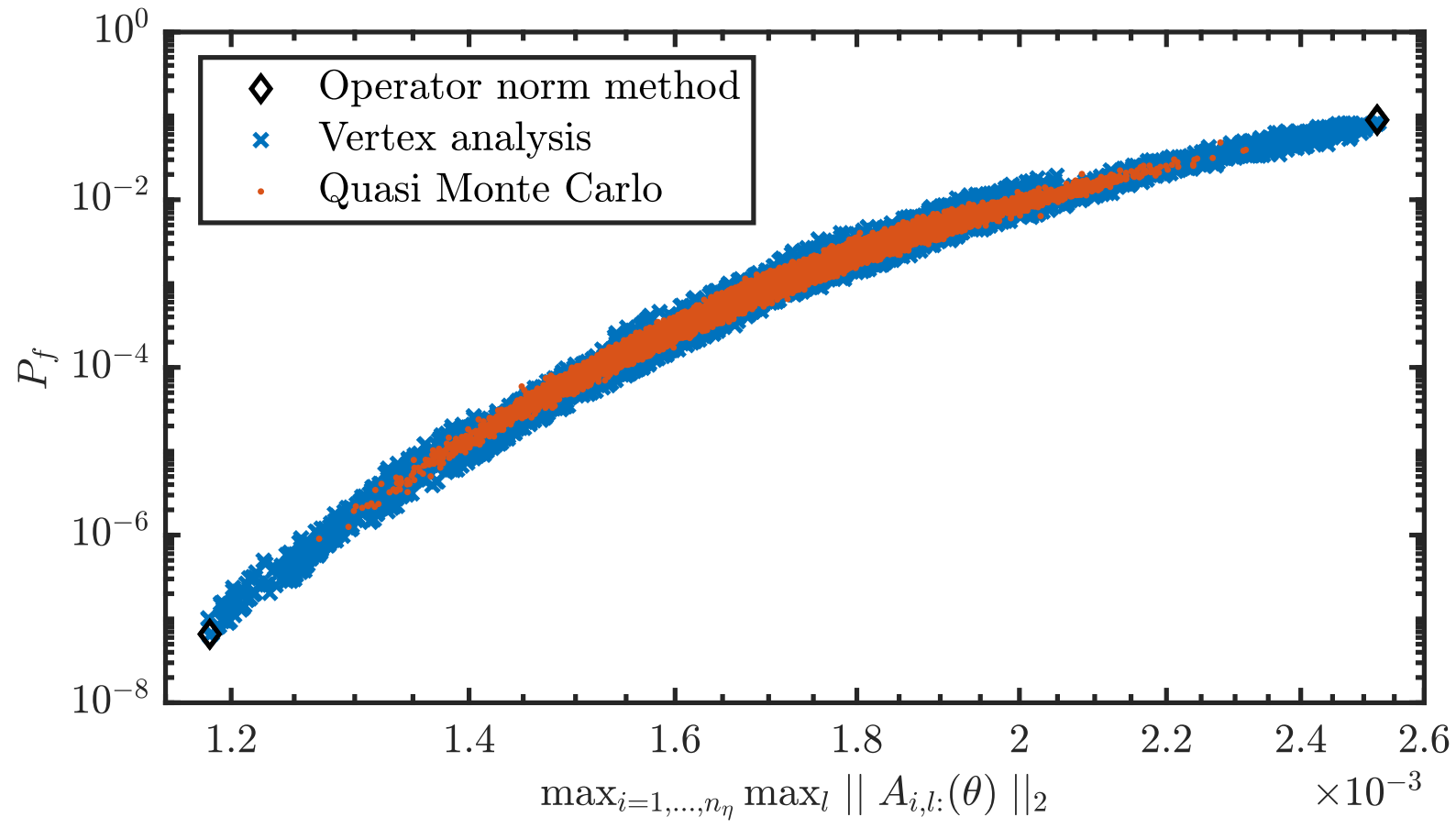

Figure 3: Results of the propagation of the uncertainty through the building model, obtained by performing a Vertex analysis (blue crosses), Quasi Monte Carlo sampling (orange dots) and the optimization approach (black diamonds).

\section{Conclusions}

This paper presents a new approach to efficiently and effectively bound the responses and probability of failure of a model that is affected by combinations of epistemic, aleatory and imprecise probabilistic uncertainty. Whereas such propagation is typically performed following a double-loop approach, the developments in this paper allow for propagating these sources of uncertainty with a strict decoupling between aleatory and epistemic uncertainty by virtue of the operator norm theorem. 
The paper shows that, under the specific scope of linear models subjected to epistemic uncertainty to which an imprecise probabilistic load is applied, a gain in computational efficiency with several orders of magnitude can be obtained. Two case studies highlight the effectivity and efficiency of the method, especially in comparison to naive double-loop approaches. As limitations of the method, it should be noted that (1) only linear models can be considered due to the definition of the operator norm theorem and (2) aleatory uncertainty within the linear map (i.e., the structural model) is not possible due to the non-separability of the sources of uncertainty in this case.

\section{Acknowledgements}

Matthias Faes acknowledges the financial support of the Research Foundation Flanders (FWO) in the context of his post-doctoral grant under grant number 12P3519N. Matthias Faes furthermore acknowledges the financial support of the Alexander von Humboldt foundation. Marcos Valdebenito acknowledges the support of ANID (National Agency for Research and Development, Chile) under its program FONDECYT, grant number 1180271.

\section{Appendix A. Karhunen-Loève expansion}

Assume a stochastic process $p(t, \boldsymbol{\xi})$. This process is represented at discrete time instants by means of the Karhunen-Loève expansion as:

$$
\boldsymbol{p}(\boldsymbol{\xi})=\boldsymbol{\mu}_{p}+\boldsymbol{\Psi} \boldsymbol{\Lambda}^{1 / 2} \boldsymbol{\xi},
$$

or equivalently:

$$
\boldsymbol{p}(\boldsymbol{\xi})=\boldsymbol{\mu}_{p}+\boldsymbol{B} \boldsymbol{\xi},
$$

where $\boldsymbol{p}$ denotes a $n_{T} \times 1$ vector containing the sample of the loading; $n_{T}$ is the total number of time steps, which is equal to $n_{T}=T / \Delta t+1 ; \boldsymbol{\xi}$ is a realisation of the random variable vector $\boldsymbol{\Xi}$ which follows a $n_{K L}$-dimensional standard Gaussian distribution; $n_{K L}$ is the number of terms retained in the KL expansion; $\Psi$ is a $n_{T} \times n_{K L}$ matrix whose columns contain the eigenvectors associated with the largest $n_{K L}$ eigenvalues of the discrete autocovariance matrix $\boldsymbol{\Gamma}$ of the Gaussian process; $\boldsymbol{\mu}_{p}$ is the mean of the stochastic process; $\boldsymbol{\Lambda}$ is a $n_{K L} \times n_{K L}$ matrix whose diagonal contains the largest $n_{K L}$ eigenvalues of the covariance matrix of the stochastic process and $\boldsymbol{B}$ a matrix collecting the eigenvectors scaled by the square root of the eigenvalues of the covariance matrix. As is clear from this formulation, in case the central moments of the process become interval valued, a definition of the process of clearly separated aleatory and epistemic parameters is obtained in an affine form. 


\section{Appendix B. Clough-Penzien spectrum}

One of the most commonly used parametric models for the power spectral density associated with ground acceleration is the Kanai-Tajimi spectrum (see, e.g. [52]), whose physical basis consists of a white noise process of spectral intensity $S_{0}$ associated with the bedrock excitation that passes through a linear soil filter characterized in terms of a natural frequency $\omega_{g}$ and damping $\zeta_{g}$. A drawback of the Kanai-Tajimi spectrum is that its associated velocity and displacement power spectra are not defined as the circular frequency tends to zero $(\omega \rightarrow 0)$. Such issue is remedied by the Clough-Penzien power spectrum, which passes the signal produced by the Kanai-Tajimi spectrum through an additional linear filter with natural frequency $\omega_{f}$ and damping $\zeta_{f}$. The expression for the Clough-Penzien power spectrum $S^{\mathrm{CP}}$ is given by $[52,53]$ :

$$
S^{\mathrm{CP}}(\omega)=\frac{\omega_{g}^{4}+\left(2 \zeta_{g} \omega_{g} \omega\right)^{2}}{\left(\omega_{g}^{2}-\omega^{2}\right)^{2}+\left(2 \zeta_{g} \omega_{g} \omega\right)^{2}} \cdot \frac{\omega^{4}}{\left(\omega_{f}^{2}-\omega^{2}\right)^{2}+\left(2 \zeta_{f} \omega_{f} \omega\right)^{2}} \cdot S_{0}
$$

Typical values for the filter parameters associated with the Clough-Penzien power spectrum as suggested in [54] are shown in Table B.5. The autocorrelation function $R^{C P}(\tau)$ associated with the Clough-Penzien

\begin{tabular}{|c|c|c|c|c|}
\hline Soil type & $\omega_{g}[\mathrm{rad} / \mathrm{s}]$ & $\zeta_{g}$ & $\omega_{f}[\mathrm{rad} / \mathrm{s}]$ & $\zeta_{f}$ \\
\hline Firm & $8 \pi$ & 0.60 & $0.8 \pi$ & 0.60 \\
\hline Medium & $5 \pi$ & 0.60 & $0.5 \pi$ & 0.60 \\
\hline Soft & $2.4 \pi$ & 0.85 & $0.24 \pi$ & 0.85 \\
\hline
\end{tabular}

Table B.5: Filter parameters associated with Clough-Penzien power spectrum

power spectrum is calculated taking the inverse Fourier transform of $S^{\mathrm{CP}}$; the reader is referred to [55] for the exact formulations. The above discussion assumes that the ground acceleration can be modeled as a wide-sense stationary stochastic process. It is clear that this is a simplifying assumption, as ground acceleration exhibits a non stationary behavior. A possible means for including such effect in the CloughPenzien model consists of modulating the white noise bedrock process by means of a deterministic function of time $m(t)$ (see, e.g. [56]). Here, the so-called Shinozuka and Sato modulating function [57] is considered:

$$
m(t)=\frac{1}{c_{3}}\left(e^{-c_{1} t}-e^{-c_{2} t}\right)
$$

where $c_{1}$ and $c_{2}$ are parameters of the model and $c_{3}$ is defined such that the maximum value of the modulating function is equal to unity, yielding:

$$
c_{3}=\frac{c_{1}}{c_{2}-c_{1}} e^{\frac{c_{2}}{c_{2}-c_{1}} \ln \left(\frac{c_{2}}{c_{1}}\right)}
$$




\section{Appendix C. Calculation of the response of a linear structure by means of the convolution integral}

Assume that the response $y(t, \boldsymbol{\xi})$ of a linear structure corresponds to the dynamic displacement of a certain degree-of-freedom, where the dynamics of the structure are represented by:

$$
\boldsymbol{M} \ddot{\boldsymbol{\eta}}(t, \boldsymbol{\xi})+\boldsymbol{C} \dot{\boldsymbol{\eta}}(t, \boldsymbol{\xi})+\boldsymbol{K} \boldsymbol{\eta}(t, \boldsymbol{\xi})=\boldsymbol{\rho} p(t, \boldsymbol{\xi}),
$$

with $t \in[0, T]$ and $\boldsymbol{\eta}(0, \boldsymbol{\xi})=\dot{\boldsymbol{\eta}}(0, \boldsymbol{\xi})=\mathbf{0}$; and where $t$ denotes time, $\boldsymbol{\eta} \in \mathbb{R}^{n_{D}}, \dot{\boldsymbol{\eta}} \in \mathbb{R}^{n_{D}}$ and $\ddot{\boldsymbol{\eta}} \in \mathbb{R}^{n_{D}}$ are vectors that represent the displacement, velocity and acceleration; $\boldsymbol{M} \in \mathbb{R}^{n_{D} \times n_{D}}, \boldsymbol{C} \in \mathbb{R}^{n_{D} \times n_{D}}$, and $\boldsymbol{K} \in \mathbb{R}^{n_{D} \times n_{D}}$ are the mass, (classical) damping and stiffness matrices. The stochastic Gaussian loading $p(t, \boldsymbol{\xi})$ is coupled to the degrees-of-freedom of the structure by means of vector $\boldsymbol{\rho}$.

Such response can be calculated by means of the convolution integral:

$$
y(t, \boldsymbol{\xi})=\int_{0}^{t} h(t-\tau) p(t, \boldsymbol{\xi}) d \tau
$$

where $h(t)$ is the unit impulse response function, which is defined as:

$$
h(t)=\sum_{v=1}^{n_{D}} \frac{\gamma^{T} \boldsymbol{\phi}_{v} \boldsymbol{\phi}_{v}^{T}}{\boldsymbol{\phi}_{v}^{T} \boldsymbol{M} \boldsymbol{\phi}_{v}} \frac{1}{\omega_{v, d}} e^{-\zeta_{v} \omega_{v} t} \sin \left(\omega_{v, d} t\right),
$$

where $\phi_{v}, v=1, \ldots, n_{D}$ are the eigenvectors associated with the eigenproblem of the undamped equation of motion; $\omega_{v}, v=1, \ldots, n_{D}$ are the natural frequencies of the system; $\zeta_{v}, v=1, \ldots, n_{D}$ are the corresponding damping ratios; $\omega_{d, v}=\omega_{v} \sqrt{\left(1-\zeta_{v}^{2}\right)}, v=1, \ldots, n_{D}$ are the damped frequencies; $\gamma$ is a vector that retrieves the degree-of-freedom of interest; and (. $)^{T}$ denotes transpose.

Taking into account the representation of the stochastic loading in terms of the Karhunen-Loève expansion as described in Appendix A and time discretization step $\Delta t$, the convolution integral in Eq. (C.2) can be approximated as a summation, where the dynamic response of interest evaluated at time $t_{k}$ is:

$$
\begin{aligned}
y\left(t_{k}, \boldsymbol{\xi}\right) & =\sum_{l_{1}=1}^{k} \Delta t \epsilon_{l_{1}} h\left(t_{k}-t_{l_{1}}\right)\left(\sum_{l_{2}=1}^{n_{K L}} \psi_{l_{1}, l_{2}} \sqrt{\lambda_{l_{2}}} \xi_{l_{2}}\right) \\
& =\boldsymbol{a}_{k}^{T} \boldsymbol{\xi}, \quad k=1, \ldots, n_{T}
\end{aligned}
$$


where $\psi_{l_{1}, l_{2}}$ is the $\left(l_{1}, l_{2}\right)$-th element of matrix $\boldsymbol{\Psi} ; \boldsymbol{a}_{k}$ is a vector of dimension $n_{K L} \times 1$ such that:

$$
\boldsymbol{a}_{k}=\left[\begin{array}{c}
\sum_{l_{1}=1}^{k} \Delta t \epsilon_{l_{1}} h\left(t_{k}-t_{l_{1}}\right) \psi_{l_{1}, 1} \sqrt{\lambda_{1}} \\
\sum_{l_{1}=1}^{k} \Delta t \epsilon_{l_{1}} h\left(t_{k}-t_{l_{1}}\right) \psi_{l_{1}, 2} \sqrt{\lambda_{2}} \\
\vdots \\
\sum_{l_{1}=1}^{k} \Delta t \epsilon_{l_{1}} h\left(t_{k}-t_{l_{1}}\right) \psi_{l_{1}, n_{K L}} \sqrt{\lambda_{n_{K L}}}
\end{array}\right]
$$

and $\epsilon_{l_{1}}$ is a coefficient depending on the numerical integration scheme used in the evaluation of the convolution integral. For the case where the trapezoidal integration rule is chosen, $\epsilon_{l_{1}}=1 / 2$ if $l_{1}=1$ or $l_{1}=k$; otherwise, $\epsilon_{l_{1}}=1$.

\section{References}

[1] S. Bi, M. Broggi, P. Wei, M. Beer, The Bhattacharyya distance: Enriching the P-box in stochastic sensitivity analysis, Mechanical Systems and Signal Processing 129 (2019) 265-281. doi:10.1016/j.ymssp.2019.04.035.

[2] D. Moens, M. Hanss, Non-probabilistic finite element analysis for parametric uncertainty treatment in applied mechanics: Recent advances, Finite Elements in Analysis and Design 47 (2011) 4-16. doi:10.1016/j.finel.2010.07.010.

[3] R. E. Moore, Methods and applications of interval analysis, SIAM, 1979.

[4] M. Faes, D. Moens, Recent Trends in the Modeling and Quantification of Non-probabilistic Uncertainty, Archives of Computational Methods in Engineering (2019). doi:10.1007/s11831-019-09327-x.

[5] L. G. Crespo, B. K. Colbert, S. P. Kenny, D. P. Giesy, On the quantification of aleatory and epistemic uncertainty using Sliced-Normal distributions, Systems and Control Letters 134 (2019) 104560. doi:10.1016/j.sysconle.2019.104560.

[6] M. Imholz, M. Faes, D. Vandepitte, D. Moens, Robust uncertainty quantification in structural dynamics under scarse experimental modal data: A Bayesian-interval approach, Journal of Sound and Vibration 467 (2020) 114983. doi:10.1016/j.jsv.2019.114983.

[7] M. Broggi, M. Faes, E. Patelli, Y. Govers, D. Moens, M. Beer, Comparison of Bayesian and interval uncertainty quantification: Application to the AIRMOD test structure, in: 2017 IEEE Symposium Series on Computational Intelligence, SSCI 2017 - Proceedings, volume 2018-Janua, 2018, pp. 1-8. doi:10.1109/SSCI.2017.8280882. 
[8] M. Faes, M. Broggi, E. Patelli, Y. Govers, J. Mottershead, M. Beer, D. Moens, A multivariate interval approach for inverse uncertainty quantification with limited experimental data, Mechanical Systems and Signal Processing 118 (2019) 534-548. doi:10.1016/j.ymssp.2018.08.050.

[9] M. Faes, D. Moens, On auto- and cross-interdependence in interval field finite element analysis, International Journal for Numerical Methods in Engineering 121 (2020) 2033-2050. doi:10.1002/nme.6297.

[10] M. Faes, D. Moens, Multivariate dependent interval finite element analysis via convex hull pair constructions and the Extended Transformation Method, Computer Methods in Applied Mechanics and Engineering 347 (2019) 85-102. doi:10.1016/j.cma.2018.12.021.

[11] A. Sofi, E. Romeo, O. Barrera, A. Cocks, An interval finite element method for the analysis of structures with spatially varying uncertainties, Advances in Engineering Software 128 (2019) 1-19. doi:10.1016/j.advengsoft.2018.11.001.

[12] C. Jiang, X. Han, G. Y. Lu, J. Liu, Z. Zhang, Y. C. Bai, Correlation analysis of non-probabilistic convex model and corresponding structural reliability technique, Computer Methods in Applied Mechanics and Engineering 200 (2011) 2528-2546. doi:10.1016/j.cma.2011.04.007.

[13] G. I. Schuëller, H. J. Pradlwarter, Uncertain linear systems in dynamics: Retrospective and recent developments by stochastic approaches, Engineering Structures 31 (2009) 2507-2517. doi:10.1016/j.engstruct.2009.07.005.

[14] M. B. Giles, Multilevel Monte Carlo methods, Acta Numerica 24 (2015) 259-328. doi:10.1017/S096249291500001X.

[15] S.-K. Au, J. L. Beck, Estimation of small failure probabilities in high dimensions by subset simulation, Probabilistic Engineering Mechanics 16 (2001) 263-277. doi:https://doi.org/10.1016/S02668920(01)00019-4.

[16] S. K. Au, J. L. Beck, First excursion probabilities for linear systems by very efficient importance sampling, Probabilistic Engineering Mechanics 16 (2001) 193-207. doi:10.1016/S0266-8920(01)00002-9.

[17] M. A. Misraji, M. A. Valdebenito, H. A. Jensen, C. F. Mayorga, Application of directional importance sampling for estimation of first excursion probabilities of linear structural systems subject to stochastic Gaussian loading, Mechanical Systems and Signal Processing 139 (2020) 106621. doi:10.1016/j.ymssp.2020.106621.

[18] G. Blatman, B. Sudret, Adaptive sparse polynomial chaos expansion based on least angle regression, Journal of Computational Physics 230 (2011) 2345-2367. doi:https://doi.org/10.1016/j.jcp.2010.12.021. 
[19] P. Wei, X. Zhang, M. Beer, Adaptive experiment design for probabilistic integration, Computer Methods in Applied Mechanics and Engineering 365 (2020) 113035. doi:https://doi.org/10.1016/j.cma.2020.113035.

[20] P. D. Spanos, L. M. Vargas Loli, A statistical approach to generation of design spectrum compatible earthquake time histories, International Journal of Soil Dynamics and Earthquake Engineering 4 (1985) 2-8. doi:10.1016/0261-7277(85)90029-4.

[21] I. P. Mitseas, I. A. Kougioumtzoglou, A. Giaralis, M. Beer, A novel stochastic linearization framework for seismic demand estimation of hysteretic $\{\mathrm{MDOF}\}$ systems subject to linear response spectra, Structural Safety 72 (2018) 84-98. doi:https://doi.org/10.1016/j.strusafe.2017.12.008.

[22] G. Stefanou, The stochastic finite element method: Past, present and future, Computer Methods in Applied Mechanics and Engineering 198 (2009) 1031-1051. doi:10.1016/j.cma.2008.11.007.

[23] W. Graf, M. Götz, M. Kaliske, Analysis of dynamical processes under consideration of polymorphic uncertainty, Structural Safety 52, Part B (2015) 194201. URL: http://www.sciencedirect.com/science/article/pii/S0167473014000861. doi:http://dx.doi.org/10.1016/j.strusafe.2014.09.003, Engineering Analyses with Vague and Imprecise Information.

[24] V. Kreinovich, S. A. Ferson, A new Cauchy-based black-box technique for uncertainty in risk analysis, Reliability Engineering and System Safety 85 (2004) 267-279. doi:10.1016/j.ress.2004.03.016.

[25] J. C. Helton, J. D. Johnson, W. L. Oberkampf, An exploration of alternative approaches to the representation of uncertainty in model predictions, Reliability Engineering and System Safety 85 (2004) 39-71. doi:10.1016/j.ress.2004.03.025.

[26] D. Dubois, H. Prade, Possibility theory: an approach to computerized processing of uncertainty, Springer Science \& Business Media, 2012.

[27] B. Möller, B. Michael, Fuzzy Randomness: Uncertainty in Civil Engineering and Computational Mechanics, 2004.

[28] R. Schöbi, B. Sudret, Structural reliability analysis for p-boxes using multilevel meta-models, $\quad$ Probabilistic Engineering Mechanics $48 \quad$ (2017) 27-38. doi:https://doi.org/10.1016/j.probengmech.2017.04.001. 
[29] H. B. Liu, C. Jiang, Z. Xiao, Efficient uncertainty propagation for parameterized p-box using sparsedecomposition-based polynomial chaos expansion, Mechanical Systems and Signal Processing 138 (2020) 106589. doi:10.1016/j.ymssp.2019.106589.

[30] J. Sadeghi, M. de Angelis, E. Patelli, Robust propagation of probability boxes by interval predictor models, Structural Safety 82 (2020) 101889. doi:https://doi.org/10.1016/j.strusafe.2019.101889.

[31] J. Zhang, M. D. Shields, On the quantification and efficient propagation of imprecise probabilities resulting from small datasets, Mechanical Systems and Signal Processing 98 (2018) 465-483. doi:10.1016/j.ymssp.2017.04.042.

[32] P. Wei, J. Song, S. Bi, M. Broggi, M. Beer, Z. Lu, Z. Yue, Non-intrusive stochastic analysis with parameterized imprecise probability models: I. Performance estimation, Mechanical Systems and Signal Processing 124 (2019) 349-368.

[33] A. Sofi, G. Muscolino, F. Giunta, Propagation of uncertain structural properties described by imprecise Probability Density Functions via response surface method, Probabilistic Engineering Mechanics 60 (2020). doi:10.1016/j.probengmech.2020.103020.

[34] M. Mäck, M. Hanss, Efficient Possibilistic Uncertainty Analysis of a Car Crash Scenario Using a Multifidelity Approach, ASCE-ASME J Risk and Uncert in Engrg Sys Part B Mech Engrg 5 (2019). doi:10.1115/1.4044243.

[35] H. Zhang, H. Dai, M. Beer, W. Wang, Structural reliability analysis on the basis of small samples: An interval quasi-Monte Carlo method, Mechanical Systems and Signal Processing 37 (2013) 137-151. URL: http://dx.doi.org/10.1016/j.ymssp. 2012.03.001. doi:10.1016/j.ymssp.2012.03.001.

[36] H. Zhang, Interval importance sampling method for finite element-based structural reliability assessment under parameter uncertainties, Structural Safety 38 (2012) 1-10. URL: http://dx.doi.org/10.1016/j.strusafe.2012.01.003. doi:10.1016/j.strusafe.2012.01.003.

[37] C. Wang, H. Zhang, M. Beer, Computing tight bounds of structural reliability under imprecise probabilistic information, Computers and Structures 208 (2018) 92-104. URL: https://doi.org/10.1016/j.compstruc.2018.07.003. doi:https://doi.org/10.1016/j.compstruc.2018.07.003.

[38] L. Sun, H. Alkhatib, B. Kargoll, V. Kreinovich, I. Neumann, Ellipsoidal and Gaussian Kalman filter model for discrete-time nonlinear systems, Mathematics 7 (2019) 1-22. doi:10.3390/MATH7121168. 
[39] M. Beer, S. Ferson, V. Kreinovich, Imprecise probabilities in engineering analyses, Mechanical Systems and Signal Processing 37 (2013) 4-29. doi:10.1016/j.ymssp.2013.01.024.

[40] C. Jiang, J. Zheng, X. Han, Probability-interval hybrid uncertainty analysis for structures with both aleatory and epistemic uncertainties: a review, Structural and Multidisciplinary Optimization 57 (2018) 2485-2502. doi:10.1007/s00158-017-1864-4.

[41] F. Leichsenring, C. Jenkel, W. Graf, M. Kaliske, Numerical simulation of wooden structures with polymorphic uncertainty in material properties, International Journal of Reliability and Safety 12 (2018) 24-45. doi:10.1056/NEJMoa1716816.

[42] M. Fina, P. Weber, W. Wagner, Polymorphic uncertainty modeling for the simulation of geometric imperfections in probabilistic design of cylindrical shells, Structural Safety 82 (2020) 101894. doi:10.1016/j.strusafe.2019.101894.

[43] W. Gao, D. Wu, K. Gao, X. Chen, F. Tin-loi, Structural reliability analysis with imprecise random and interval fields, Applied Mathematical Modelling 55 (2018) 49-67. doi:10.1016/j.apm.2017.10.029.

[44] S. Freitag, B. T. Cao, J. Ninić, G. Meschke, Recurrent neural networks and proper orthogonal decomposition with interval data for real-time predictions of mechanised tunnelling processes, Computers and Structures 207 (2018) 258-273. doi:10.1016/j.compstruc.2017.03.020.

[45] M. Faes, M. A. Valdebenito, D. Moens, M. Beer, Bounding the First Excursion Probability of Linear Structures Subjected to Imprecise Stochastic Loading, preprint submitted to elsevier - preprint availabe via ResearchGate (2020).

[46] G. I. Schuëller, H. J. Pradlwarter, Benchmark study on reliability estimation in higher dimensions of structural systems - an overview, Structural Safety 29 (2007) 167-182.

[47] M. Faes, D. Moens, Imprecise random field analysis with parametrized kernel functions, Mechanical Systems and Signal Processing 134 (2019) 106334. doi:10.1016/j.ymssp.2019.106334.

[48] J. A. Tropp, Topics in Sparse Approximation, Ph.D. thesis, The University of Texas at Austin, 2004. doi:10.1109/TIT.2004.834793.

[49] M. G. R. Faes, M. A. Valdebenito, Fully decoupled reliability-based design optimization of structural systems subject to uncertain loads, Computer Methods in Applied Mechanics and Engineering 371 (2020) 113313. doi:10.1016/j.cma.2020.113313. 
[50] C. K. Monson, K. D. Seppi, Bayesian optimization models for particle swarms, in: Proceedings of the 7th Annual Conference on Genetic and Evolutionary Computation, GECCO '05, Association for Computing Machinery, New York, NY, USA, 2005, p. 193-200. doi:10.1145/1068009.1068039.

[51] E. Patelli, H. M. Panayirci, M. Broggi, B. Goller, P. Beaurepaire, H. J. Pradlwarter, G. I. Schuëller, General purpose software for efficient uncertainty management of large finite element models, Finite Elements in Analysis and Design 51 (2012) 31-48. doi:10.1016/j.finel.2011.11.003.

[52] T. T. Soong, M. Grigoriu, Random Vibration of Mechanical and Structural Systems, Prentice Hall, Englewood Cliffs, New Jersey, 1993.

[53] A. Zerva, Spatial Variation of Seismic Ground Motions - Modeling and Engineering Applications, CRC Press, 2009.

[54] G. Deodatis, Non-stationary stochastic vector processes: seismic ground motion applications, Probabilistic Engineering Mechanics 11 (1996) 149-167. doi:https://doi.org/10.1016/0266-8920(96)00007-0.

[55] G. Fu, Seismic response statistics of $\{\mathrm{SDOF}\}$ system to exponentially modulated coloured input: An explicit solution, Earthquake Engineering \& Structural Dynamics 24 (1995) 1355-1370. doi:10.1002/eqe.4290241006.

[56] C.-H. Yeh, Y. K. Wen, Modeling of nonstationary ground motion and analysis of inelastic structural response, Structural Safety 8 (1990) 281-298.

[57] M. Shinozuka, Y. Sato, Simulation of nonstationary random process, Journal of the Engineering Mechanics Division 93 (1967) 11-40. 